\title{
Tribaryons in a constituent quark model
}

\author{
Aaron Park $\odot^{*}$ and Su Houng Lee ${ }^{\dagger}$ \\ Department of Physics and Institute of Physics and Applied Physics, Yonsei University, Seoul 03722, Korea
}

(Received 21 September 2020; accepted 28 October 2020; published 24 November 2020)

\begin{abstract}
We calculate the matrix elements of the color-spin interaction for all possible multiquark states of tribaryons in flavor SU(3) broken case. For that purpose, we construct the flavor $\otimes$ color $\otimes$ spin wave functions of the tribaryons, which are taken to be antisymmetric to satisfy the Pauli exclusion principle. Furthermore, we analyze the diquark structure of the tribaryon configurations using the symmetric and antisymmetric basis set of flavor, color, and spin states.
\end{abstract}

DOI: 10.1103/PhysRevD.102.096024

\section{INTRODUCTION}

While the interactions between quarks and gluons are described by QCD, which is based on the color SU(3) gauge group, the phenomenological interaction between constituent quarks within a hadron can be taken to be mediated by gluons [1] or mesons [2]. The constituent quark models can well reproduce the mass of normal hadrons, which are composed of three quarks for baryons and a quark-antiquark pair for mesons. On the other hand, ever since Jaffe suggested the possibility of a stable dibaryon [3] and tetraquark using the bag model [4,5], exotic hadrons consisting of more than three quarks or quark-antiquark have been a subject of experimental as well as theoretical studies (for a recent review, see Ref. [6]). The quark interaction within a multiquark configuration is the central theme in understanding exotic multiquark configuration within the constituent quark model [7].

Multiquark configurations and their quark interactions can also be used to understand the baryon-baryon interaction as was first attempted successfully within the quark-cluster model [8]. In fact, it was recently shown that the shortdistance part of the baryon-baryon interaction including those with strangeness extracted from the recent lattice calculation [9] can be well reproduced using the constituent quark model [10]. The crucial input in understanding the flavor dependence in these interactions is the color-spin interaction between quarks and the antisymmetrized flavor $\otimes$ color $\otimes$ spin wave functions that is required by the Pauli principle $[8,10]$.

As for multiquark configuration involving three baryons, only a limited number of works exist [11-15]. The tribaryon

\footnotetext{
*aaron.park@yonsei.ac.kr

'suhoung@yonsei.ac.kr
}

Published by the American Physical Society under the terms of the Creative Commons Attribution 4.0 International license. Further distribution of this work must maintain attribution to the author(s) and the published article's title, journal citation, and DOI. Funded by SCOAP ${ }^{3}$. configuration is related to the three-nucleon interaction at short distance [14-16], whose strength is important in relation to providing the stability of nuclear matter at high density. For example, the intrinsic three-body nuclear force including hyperons has been shown to provide a solution to the hyperon puzzle in a neutron star $[17,18]$. In a previous work, we have shown that the intrinsic three-body forces vanish in the flavor $\mathrm{SU}(3)$ symmetric case, undermining the solution for the hyperon puzzle [15]. However, it is important to note that the situation might change if the $\mathrm{SU}(3)$ symmetry breaking effect is taken into account. The importance of the $\mathrm{SU}(3)$ breaking effect cannot be overemphasized in the quark model. While the color-spin interaction is large for the H-dibaryon in the $\mathrm{SU}(3)$ symmetric limit, the strength drastically reduces when the symmetry breaking is introduced [19].

In this paper, we will calculate the expectation values of the color-spin interaction of a tribaryon using a constituent quark model in the flavor SU(3) breaking case. In particular, for each tribaryon configuration, we will explicitly calculate the flavor $\otimes$ color $\otimes$ spin wave functions and analyze the diquark configurations in them. This is accomplished by identifying the possible diquark configuration for all quark pairs within the tribaryon system and calculating the probability of each diquark configuration within the total wave function. Through our study, we will elucidate the importance of the flavor SU(3) breaking effect for each tribaryon configuration and highlight the important diquark configuration for attractive tribaryon configurations.

This paper is organized as follows. In Sec. II, we introduce the form of color-spin interaction and a parameter $\delta$ which can describe the flavor SU(3) breaking effect. In Sec. III, we represent in detail the method for constructing the wave function of a tribaryon. In Sec. IV, we show the color-spin matrix elements of the tribaryons for all possible quantum numbers in the flavor SU(3) broken case. In Sec. V, we calculate the static binding energy of a tribaryon for the lowest flavor channels for each spin. In Sec. VI, we analyze the diquark structures of the tribaryons using symmetric and 
antisymmetric basis set of flavor, color, and spin wave function. Finally, Sec. VII is devoted to a summary and concluding remarks.

\section{COLOR-SPIN INTERACTION}

In this work, we present the matrix elements of color-spin interaction for the tribaryons in the flavor $\mathrm{SU}(3)$ broken case. The color-spin interaction is defined as

$$
V_{C S}=-\sum_{i<j}^{9} \frac{1}{m_{i} m_{j}} \lambda_{i}^{c} \lambda_{j}^{c} \sigma_{i} \cdot \sigma_{j} \equiv \frac{1}{m_{u} m_{u}} H_{C S},
$$

where $\lambda_{i}^{c}, m_{i}$, and $m_{u}$ are, respectively, the color $\mathrm{SU}(3)$ Gell-Mann matrices, the constituent quark mass of the $i$ th quark, and the constituent quark mass of $u, d$ quarks. To analyze the flavor SU(3) breaking effect, it is useful to introduce the following strange quark mass parameter:

$$
\delta=1-\frac{m_{u}}{m_{s}} .
$$

When the mass of the strange quark becomes the same as that of the $u, d$ quarks, the flavor $\mathrm{SU}(3)$ breaking effect vanishes. For a totally antisymmetric flavor $\otimes$ color $\otimes$ spin wave function with a given flavor $\mathrm{SU}(N)$, we can easily calculate the expectation value of the color-spin interaction using the following formula without considering the explicit form of the wave function,

$$
\begin{aligned}
-\sum_{i<j}^{n} \lambda_{i}^{c} \lambda_{j}^{c} \sigma_{i} \cdot \sigma_{j} & =\frac{(N+6)}{3 N} n^{2}+(-2 N-4) n+\frac{4}{3} S(S+1)+4 C_{F}^{\mathrm{SU}(N)}+2 C_{C}, \\
4 C_{F}^{\mathrm{SU}(N)} & =\frac{2 m}{N}\left(N^{2}-1\right)+\frac{4}{N}(N-1) \sum_{i=1}^{N-1}\left(\begin{array}{c}
\sum_{j=1}^{i} p_{N-j} \\
2
\end{array}\right)-4 \sum_{i=1}^{N-2}\left[\sum_{j=i+1}^{N-1}(j-i) p_{j}\left(1+\frac{1}{N} \sum_{k=i}^{N-1} p_{k}\right)\right],
\end{aligned}
$$

where $n$ is the total number of quarks; $C_{F}^{\mathrm{SU}(N)}=\frac{1}{4} \lambda^{F} \lambda^{F}$ and $C_{C}$ are the first kind of the Casimir operator of flavor $\mathrm{SU}(N)$ and color $\mathrm{SU}(3)$, respectively; $p_{i}$ is the number of columns containing $i$ boxes in a column in a Young diagram; and $m$ is the total number of boxes minus $N p_{N}$, which is $\sum_{k=1}^{N-1} k p_{k}$. For the flavor $\mathrm{SU}(3)$ symmetric case, Eq. (3) reduces to the form

$$
\begin{aligned}
-\sum_{i<j}^{n} \lambda_{i}^{c} \lambda_{j}^{c} \sigma_{i} \cdot \sigma_{j} & =n(n-10)+\frac{4}{3} S(S+1)+4 C_{F}+2 C_{C}, \\
4 C_{F} & =\frac{4}{3}\left(p_{1}^{2}+p_{2}^{2}+3 p_{1}+3 p_{2}+p_{1} p_{2}\right),
\end{aligned}
$$

where $C_{F}$ is the first kind of the Casimir operator of flavor $\mathrm{SU}(3)$. Then, we can check that the matrix element of
Eq. (3) becomes the same as the flavor SU(3) symmetric case when $N=3$.

\section{WAVE FUNCTION OF A TRIBARYON}

In this work, we limit our discussion to the case where the orbital par of the quark wave function is totally symmetric. This means that the remaining flavor $\otimes$ color $\otimes$ spin part of the wave function should be antisymmetric to satisfy the Pauli exclusion principle. In general, if we consider the tribaryon as the sum of three baryon states, the orbital part does not have to be totally symmetric. We represent the possible orbital state of a tribaryon in Appendix for future work.

For the totally symmetric orbital state, there are eight possible flavor states for the tribaryons given as follows [14]:

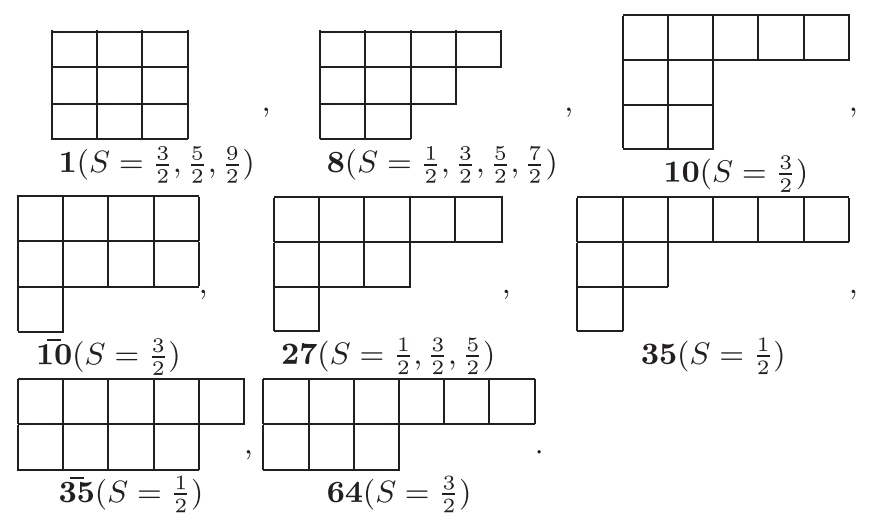


The brackets below each flavor state show the possible spin states. However, in the flavor SU(3) broken case, the number of possible flavor states is limited in relation to isospin and strangeness. As an example, for $I=2, S=\frac{3}{2}$ and one strange quark, only one $\mathrm{SU}(3)$ flavor state contributes, which is a flavor 64 multiplet,

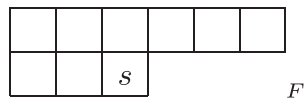

Additionally, when there is more than one strange quark, the strange quarks should not be located in the same column because their flavor wave function is symmetric. For example, the flavor 27 multiplet can accommodate the state with $I=2, S=\frac{5}{2}$ and strangeness $=-3$,

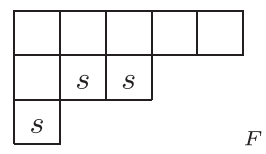

Similarly, the corresponding color $\otimes$ spin coupling state can be constructed as follows:

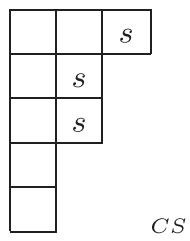

Now, using the symmetric property of Young-Yamanouchi basis [20], we can represent the above flavor and color $\otimes$ spin coupling state as follows:
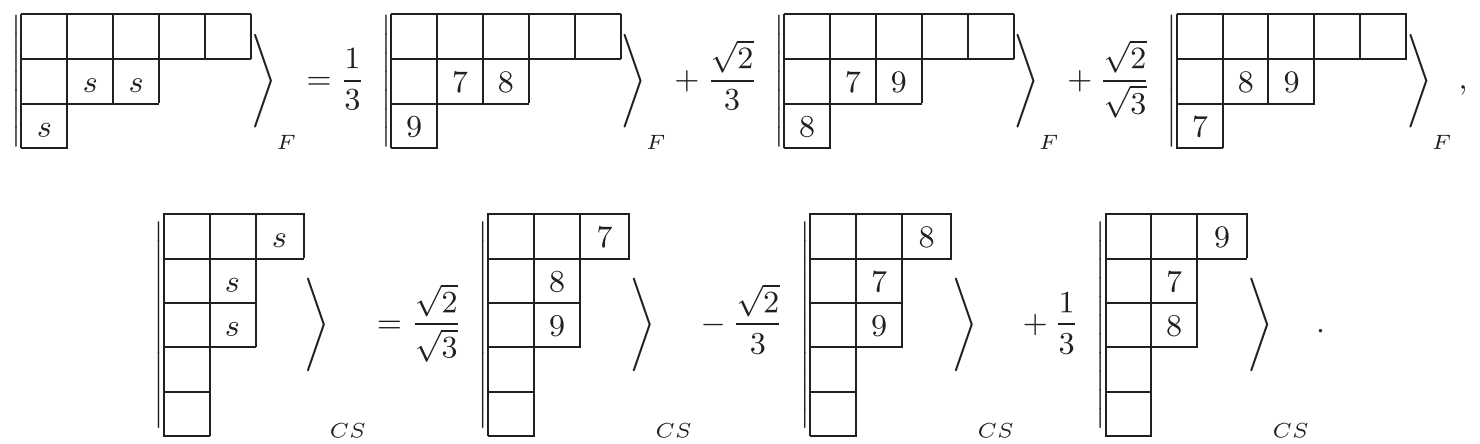

We can check that the above flavor and color $\otimes$ spin coupling states are symmetric and antisymmetric under the exchange among 7,8 , and 9 , respectively. Now, we can construct the flavor $\otimes$ color $\otimes$ spin wave function as

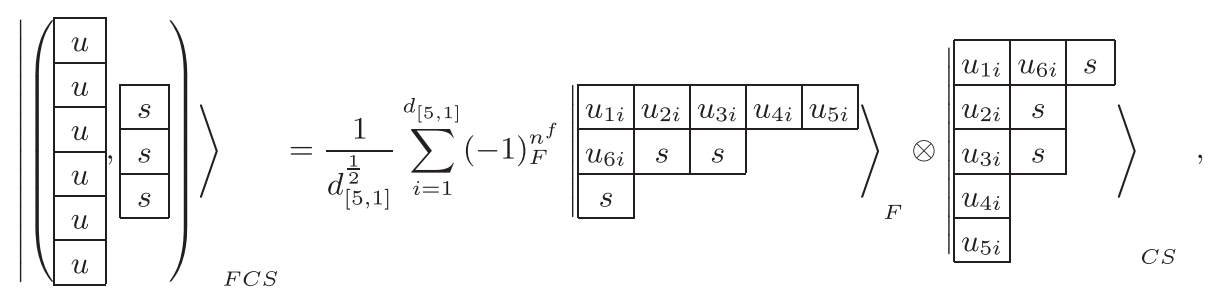

where $d_{f}$ is the dimension of the irreducible representation $f$ of $S_{6}, n_{F}^{f}$ is the number of transpositions necessary to bring the corresponding Young tableau of flavor state to a normal Young tableau [20], and $\left(u_{1 i}, u_{2 i} \ldots\right)$ is an array of numbers from 1 to 6 which can make the corresponding Young tableau.

Next, we need to decompose the color $\otimes$ spin coupling state into color and spin states using the ClebschGordan coefficients of $S_{9}$. Then, the total wave function of a tribaryon with $I=2, S=\frac{5}{2}$, and strnageness $=-3$ can be represented as 


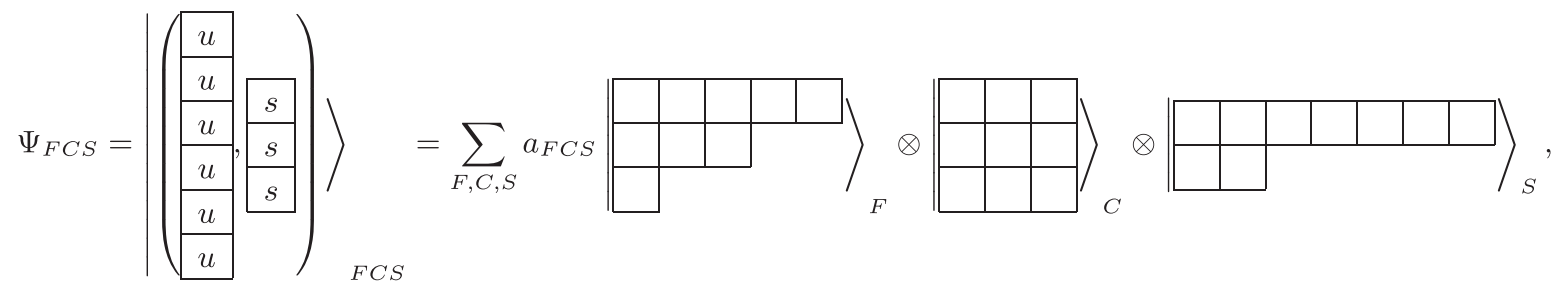

where $a_{F C S}$ are the coefficients which can be constructed from $n^{F}$, Clebsch-Gordan coefficients of $S_{9}$, and the coefficients in Eq. (5). It should be noted that the blanks on the above equation are actually filled with numbers to form a Young tableau. After representing the wave function using each Young-Yamanouchi basis, we can easily calculate the matrix elements of color-spin interaction with the formula

$$
\begin{aligned}
\lambda_{i}^{c} \lambda_{j}^{c} & =2(i j)-\frac{2}{3} I, \\
\sigma_{i} \cdot \sigma_{j} & =2(i j)-I,
\end{aligned}
$$

where $(i j)$ is the transposition between $i$ th and $j$ th quarks and $I$ is the identity matrix in the corresponding irreducible representation. In general, the generator $\lambda$ of $\mathrm{SU}(N)$ is as follows:

$$
\lambda_{i} \lambda_{j}=2(i j)-\frac{2}{N} .
$$

\section{COLOR-SPIN INTERACTION MATRIX ELEMENTS}

Here, we represent the matrix elements of color-spin interaction for all possible tribaryon states in the flavor $\mathrm{SU}(3)$ broken case. To represent the symmetric property of the wave function, we use the $\{i j k \cdots\}$ notation, which means antisymmetry under the exchange of particles among $i, j, k, \cdots$. For example, in the case of a tribaryon with three strange quarks, it should satisfy $\{123456\}\{789\}$ because we put the strange quarks on the last position of the wave function.

\section{A. $q^{8} s:\{12345678\} 9$}

(i) $I=2, S=\frac{3}{2}(F=64)$ :

$$
H_{C S}=56-\frac{16}{3} \delta
$$

(ii) $I=2, S=\frac{1}{2}(F=35)$ :

$$
H_{C S}=40+\frac{32}{3} \delta
$$

(iii) $I=1, S=\frac{5}{2}(F=27)$

$$
H_{C S}=\frac{104}{3}+\frac{16}{3} \delta
$$

(iv) $I=1, S=\frac{3}{2}(F=64,27)$ :

$$
H_{C S}=\left(\begin{array}{cc}
56-\frac{368}{21} \delta & \frac{16 \sqrt{10}}{21} \delta \\
\frac{16 \sqrt{10}}{21} \delta & 28+\frac{172}{21} \delta
\end{array}\right)
$$

(v) $I=1, S=\frac{1}{2}(F=\overline{35}, 27)$ :

$$
H_{C S}=\left(\begin{array}{cc}
40-\frac{20}{3} \delta & -\frac{4}{3} \delta \\
-\frac{4}{3} \delta & 24+\frac{28}{3} \delta
\end{array}\right)
$$

(vi) $I=0, S=\frac{3}{2}(F=\overline{10})$ :

$$
H_{C S}=20+\frac{20}{3} \delta
$$

(vii) $I=0, S=\frac{1}{2}(F=\overline{35})$ :

$$
H_{C S}=40-\frac{40}{3} \delta
$$

$$
\text { B. } \boldsymbol{q}^{7} \boldsymbol{s}^{2}:\{1234567\}\{89\}
$$

(i) $I=\frac{5}{2}, S=\frac{3}{2}(F=64)$ :

$$
H_{C S}=56-\frac{32}{3} \delta+\frac{8}{3} \delta^{2}
$$

(ii) $I=\frac{5}{2}, S=\frac{1}{2}(F=35)$ :

$$
H_{C S}=40+\frac{16}{3} \delta+\frac{8}{3} \delta^{2}
$$

(iii) $I=\frac{3}{2}, S=\frac{5}{2}(F=27)$ :

$$
H_{C S}=\frac{104}{3}-\frac{16}{3} \delta+\frac{8}{3} \delta^{2}
$$

(iv) $I=\frac{3}{2}, S=\frac{3}{2}(F=10,27,64)$ :

$$
H_{C S}=\left(\begin{array}{ccc}
20+\frac{26}{3} \delta+\frac{26}{9} \delta^{2} & -\frac{2 \sqrt{35}}{3 \sqrt{3}} \delta-\frac{2 \sqrt{5}}{3 \sqrt{21}} \delta^{2} & \frac{4 \sqrt{5}}{9 \sqrt{7}} \delta^{2} \\
-\frac{2 \sqrt{35}}{3 \sqrt{3}} \delta-\frac{2 \sqrt{5}}{3 \sqrt{21}} \delta^{2} & 28-\frac{2}{21} \delta+\frac{22}{7} \delta^{2} & -\frac{80}{21 \sqrt{3}} \delta-\frac{20}{21 \sqrt{3}} \delta^{2} \\
\frac{4 \sqrt{5}}{9 \sqrt{7}} \delta^{2} & -\frac{80}{21 \sqrt{3}} \delta-\frac{20}{21 \sqrt{3}} \delta^{2} & 56-\frac{544}{21} \delta+\frac{208}{63} \delta^{2}
\end{array}\right)
$$

(v) $I=\frac{3}{2}, S=\frac{1}{2}(F=27,35, \overline{35})$ :

$$
H_{C S}=\left(\begin{array}{ccc}
20+\frac{10}{3} \delta+3 \delta^{2} & -\frac{2}{3} \sqrt{\frac{5}{3}} \delta+\frac{1}{3} \sqrt{\frac{5}{3}} \delta^{2} & \frac{8}{3 \sqrt{3}} \delta+\frac{2}{3 \sqrt{3}} \delta^{2} \\
-\frac{2}{3} \sqrt{\frac{5}{3}} \delta+\frac{1}{3} \sqrt{\frac{5}{3}} \delta^{2} & 40-\frac{34}{3} \delta+\frac{29}{9} \delta^{2} & \frac{2 \sqrt{5}}{9} \delta^{2} \\
\frac{8}{3 \sqrt{3}} \delta+\frac{2}{3 \sqrt{3}} & \delta^{2} \frac{2 \sqrt{5}}{9} \delta^{2} & 40-\frac{40}{3} \delta+\frac{28}{9} \delta^{2}
\end{array}\right)
$$

(vi) $I=\frac{1}{2}, S=\frac{7}{2}(F=8)$ :

$$
H_{C S}=24+\frac{8}{3} \delta^{2}
$$




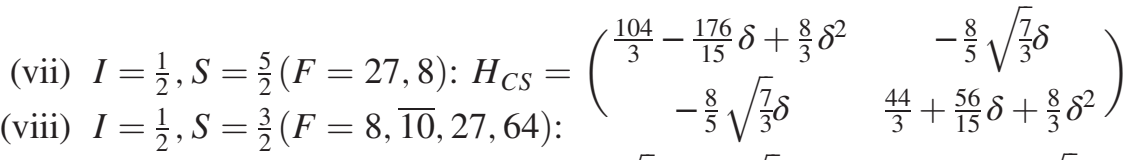

$$
H_{C S}=\left(\begin{array}{cccc}
8+\frac{52}{5} \delta+\frac{10}{3} \delta^{2} & -2 \sqrt{\frac{2}{3}} \delta-\frac{1}{3} \sqrt{\frac{2}{3}} \delta^{2} & \frac{14 \sqrt{14}}{15} \delta+\frac{1}{3} \sqrt{\frac{2}{7}} \delta^{2} & \frac{8}{3 \sqrt{21}} \delta^{2} \\
-2 \sqrt{\frac{2}{3}} \delta-\frac{1}{3} \sqrt{\frac{2}{3}} \delta^{2} & 20-\frac{10}{3} \delta+\frac{25}{9} \delta^{2} & \frac{2}{3} \sqrt{\frac{7}{3}} \delta-\frac{1}{3 \sqrt{21}} \delta^{2} & -\frac{4}{9} \sqrt{\frac{2}{7}} \delta^{2} \\
\frac{14 \sqrt{14}}{15} \delta+\frac{1}{3} \sqrt{\frac{2}{7}} \delta^{2} & \frac{2}{3} \sqrt{\frac{7}{3}} \delta-\frac{1}{3 \sqrt{21}} \delta^{2} & 28-\frac{982}{105} \delta+\frac{19}{7} \delta^{2} & -\frac{80}{21} \sqrt{\frac{2}{3}} \delta+\frac{4}{21} \sqrt{\frac{2}{3}} \delta^{2} \\
\frac{8}{3 \sqrt{21}} \delta^{2} & -\frac{4}{9} \sqrt{\frac{2}{7}} \delta^{2} & -\frac{80}{21} \sqrt{\frac{2}{3}} \delta+\frac{4}{21} \sqrt{\frac{2}{3}} \delta^{2} & 56-\frac{736}{21} \delta+\frac{200}{63} \delta^{2}
\end{array}\right)
$$

(ix) $I=\frac{1}{2}, S=\frac{1}{2}(F=8,27, \overline{35})$ :

$$
\begin{gathered}
H_{C S}=\left(\begin{array}{ccc}
4+\frac{32}{3} \delta+\frac{28}{9} \delta^{2} & -\frac{8}{3 \sqrt{3}} \delta-\frac{2}{3 \sqrt{3}} \delta^{2} & -\frac{2 \sqrt{5}}{9} \delta^{2} \\
-\frac{8}{3 \sqrt{3}} \delta-\frac{2}{3 \sqrt{3}} \delta^{2} & 24-\frac{26}{3} \delta+3 \delta^{2} & -\frac{2}{3} \sqrt{\frac{5}{3}} \delta+\frac{1}{3} \sqrt{\frac{5}{3}} \delta^{2} \\
-\frac{2 \sqrt{5}}{9} \delta^{2} & -\frac{2}{3} \sqrt{\frac{5}{3}} \delta+\frac{1}{3} \sqrt{\frac{5}{3}} \delta^{2} & 40-\frac{70}{3} \delta+\frac{29}{9} \delta^{2}
\end{array}\right) \\
\text { C. } \boldsymbol{q}^{\mathbf{6}} \boldsymbol{s}^{\mathbf{3}}:\{\mathbf{1 2 3 4 5 6}\}\{\mathbf{7 8 9}\}
\end{gathered}
$$

(i) $I=3, S=\frac{3}{2}(F=64)$ :

$$
H_{C S}=56-16 \delta+8 \delta^{2}
$$

(ii) $I=2, S=\frac{5}{2}(F=27)$ :

$$
H_{C S}=\frac{104}{3}-16 \delta+8 \delta^{2}
$$

(iii) $I=2, S=\frac{3}{2}(F=27,64)$ :

$$
H_{C S}=\left(\begin{array}{cc}
28-\frac{176}{21} \delta+\frac{178}{21} \delta^{2} & \frac{16 \sqrt{5}}{21} \delta+\frac{8 \sqrt{5}}{21} \delta^{2} \\
\frac{16 \sqrt{5}}{21} \delta+\frac{8 \sqrt{5}}{21} \delta^{2} & 56-\frac{240}{7} \delta+\frac{200}{21} \delta^{2}
\end{array}\right)
$$

(iv) $I=2, S=\frac{1}{2}(F=27,35, \overline{35})$ :

$$
H_{C S}=\left(\begin{array}{ccc}
24-\frac{8}{3} \delta+\frac{28}{3} \delta^{2} & -\frac{4}{3} \delta-\frac{2}{3} \delta^{2} & -\frac{4}{3} \delta-\frac{2}{3} \delta^{2} \\
-\frac{4}{3} \delta-\frac{2}{3} \delta^{2} & 40-20 \delta+\frac{28}{3} \delta^{2} & -\frac{2}{3} \delta^{2} \\
-\frac{4}{3} \delta-\frac{2}{3} \delta^{2} & -\frac{2}{3} \delta^{2} & 40-20 \delta+\frac{28}{3} \delta^{2}
\end{array}\right)
$$

(v) $I=1, S=\frac{7}{2}(F=8)$ :

$$
H_{C S}=24-16 \delta+8 \delta^{2}
$$

(vi) $I=1, S=\frac{5}{2}(F=8,27)$ :

$$
H_{C S}=\left(\begin{array}{cc}
\frac{44}{3}-\frac{24}{5} \delta+\frac{134}{15} \delta^{2} & \frac{8 \sqrt{14}}{15} \delta+\frac{4 \sqrt{14}}{15} \delta^{2} \\
\frac{8 \sqrt{14}}{15} \delta+\frac{4 \sqrt{14}}{15} \delta^{2} & \frac{104}{3}-\frac{368}{15} \delta+\frac{136}{15} \delta^{2}
\end{array}\right)
$$

(vii) $I=1, S=\frac{3}{2}(F=8,10, \overline{10}, 27,64)$ :

$$
H_{C S}=\left(\begin{array}{ccccc}
8-\frac{24}{5} \delta+\frac{392}{45} \delta^{2} & -2 \sqrt{\frac{2}{3}} \delta-\frac{1}{9} \sqrt{\frac{2}{3}} \delta^{2} & 2 \sqrt{\frac{2}{3}} \delta+\frac{1}{9} \sqrt{\frac{2}{3}} \delta^{2} & -\frac{28}{15} \sqrt{\frac{7}{3}} \delta-\frac{58}{15 \sqrt{21}} \delta^{2} & \frac{8}{9} \sqrt{\frac{5}{21}} \delta^{2} \\
-2 \sqrt{\frac{2}{3}} \delta-\frac{1}{9} \sqrt{\frac{2}{3}} \delta^{2} & 20-\frac{40}{3} \delta+\frac{245}{27} \delta^{2} & \frac{25}{27} \delta^{2} & -\frac{4 \sqrt{14}}{9} \delta-\frac{1}{9} \sqrt{\frac{2}{7}} \delta^{2} & -\frac{8}{27} \sqrt{\frac{10}{7}} \delta^{2} \\
2 \sqrt{\frac{2}{3}} \delta+\frac{1}{9} \sqrt{\frac{2}{3}} \delta^{2} & \frac{25}{27} \delta^{2} & 20-\frac{40}{3} \delta+\frac{245}{27} \delta^{2} & \frac{4 \sqrt{14}}{9} \delta+\frac{1}{9} \sqrt{\frac{2}{7}} \delta^{2} & \frac{8}{27} \sqrt{\frac{10}{7}} \delta^{2} \\
-\frac{28}{15} \sqrt{\frac{7}{3}} \delta-\frac{58}{15 \sqrt{21}} \delta^{2} & -\frac{4 \sqrt{14}}{9} \delta-\frac{1}{9} \sqrt{\frac{2}{7}} \delta^{2} & \frac{4 \sqrt{14}}{9} \delta+\frac{1}{9} \sqrt{\frac{2}{7}} \delta^{2} & 28-\frac{2176}{105} \delta+\frac{992}{105} \delta^{2} & \frac{80 \sqrt{5}}{63} \delta+\frac{8 \sqrt{5}}{63} \delta^{2} \\
\frac{8}{9} \sqrt{\frac{5}{21}} \delta^{2} & -\frac{8}{27} \sqrt{\frac{10}{7}} \delta^{2} & \frac{8}{27} \sqrt{\frac{10}{7}} \delta^{2} & \frac{80 \sqrt{5}}{63} \delta+\frac{8 \sqrt{5}}{63} \delta^{2} & 56-\frac{976}{21} \delta+\frac{1832}{189} \delta^{2}
\end{array}\right)
$$

(viii) $I=1, S=\frac{1}{2}(F=8,27,35, \overline{35})$ : 


$$
H_{C S}=\left(\begin{array}{cccc}
4+\frac{8}{3} \delta+\frac{254}{27} \delta^{2} & \frac{8 \sqrt{2}}{9} \delta-\frac{4 \sqrt{2}}{9} \delta^{2} & -\frac{4 \sqrt{10}}{27} \delta^{2} & -\frac{4 \sqrt{10}}{27} \delta^{2} \\
\frac{8 \sqrt{2}}{9} \delta-\frac{4 \sqrt{2}}{9} \delta^{2} & 24-\frac{56}{3} \delta+\frac{28}{3} \delta^{2} & \frac{4 \sqrt{5}}{9} \delta-\frac{2 \sqrt{5}}{9} \delta^{2} & \frac{4 \sqrt{5}}{9} \delta-\frac{2 \sqrt{5}}{9} \delta^{2} \\
-\frac{4 \sqrt{10}}{27} \delta^{2} & \frac{4 \sqrt{5}}{9} \delta-\frac{2 \sqrt{5}}{9} \delta^{2} & 40-\frac{100}{3} \delta+\frac{260}{27} \delta^{2} & -\frac{10}{27} \delta^{2} \\
-\frac{4 \sqrt{10}}{27} \delta^{2} & \frac{4 \sqrt{5}}{9} \delta-\frac{2 \sqrt{5}}{9} \delta^{2} & -\frac{10}{27} \delta^{2} & 40-\frac{100}{3} \delta+\frac{260}{27} \delta^{2}
\end{array}\right)
$$

(ix) $I=0, S=\frac{9}{2}(F=1)$ :

$$
H_{C S}=24-16 \delta+8 \delta^{2}
$$

(x) $I=0, S=\frac{7}{2}(F=8)$ :

$$
H_{C S}=24-16 \delta+8 \delta^{2}
$$

(xi) $I=0, S=\frac{5}{2}(F=1,8,27)$ :

$$
H_{C S}=\left(\begin{array}{ccc}
\frac{8}{3}-\frac{16}{9} \delta+\frac{80}{9} \delta^{2} & -\frac{8 \sqrt{35}}{9} \delta-\frac{4}{9} \sqrt{\frac{7}{5}} \delta^{2} & \frac{4}{3} \sqrt{\frac{2}{5}} \delta^{2} \\
-\frac{8 \sqrt{35}}{9} \delta-\frac{4}{9} \sqrt{\frac{7}{5}} \delta^{2} & \frac{44}{3}-\frac{664}{45} \delta+\frac{374}{45} \delta^{2} & \frac{4 \sqrt{14}}{5} \delta-\frac{2 \sqrt{14}}{15} \delta^{2} \\
\frac{4}{3} \sqrt{\frac{2}{5}} \delta^{2} & \frac{4 \sqrt{14}}{5} \delta-\frac{2 \sqrt{14}}{15} \delta^{2} & \frac{104}{3}-\frac{144}{5} \delta+\frac{44}{5} \delta^{2}
\end{array}\right)
$$

(xii) $I=0, S=\frac{3}{2}(F=1,8,27,64)$ :

$$
H_{C S}=\left(\begin{array}{cccc}
-4+\frac{8}{3} \delta+\frac{55}{6} \delta^{2} & -\frac{2 \sqrt{35}}{3} \delta-\frac{1}{3} \sqrt{\frac{7}{5}} & \delta^{2}-\frac{7}{2 \sqrt{15}} \delta^{2} & 0 \\
-\frac{2 \sqrt{35}}{3} \delta-\frac{1}{3} \sqrt{\frac{7}{5}} \delta^{2} & 8-\frac{88}{15} \delta+\frac{128}{15} \delta^{2} & -\frac{14}{5} \sqrt{\frac{7}{3}} \delta+\frac{1}{5} \sqrt{\frac{3}{7}} \delta^{2} & \frac{8}{\sqrt{105}} \delta^{2} \\
-\frac{7}{2 \sqrt{15}} \delta^{2} & -\frac{14}{5} \sqrt{\frac{7}{3}} \delta+\frac{1}{5} \sqrt{\frac{3}{7}} \delta^{2} & 28-\frac{2824}{105} \delta+\frac{1843}{210} \delta^{2} & \frac{32 \sqrt{5}}{21} \delta-\frac{16}{21 \sqrt{5}} \delta^{2} \\
0 & \frac{8}{\sqrt{105}} \delta^{2} & \frac{32 \sqrt{5}}{21} \delta-\frac{16}{21 \sqrt{5}} \delta^{2} & 56-\frac{368}{7} \delta+\frac{200}{21} \delta^{2}
\end{array}\right)
$$

(xiii) $I=0, S=\frac{1}{2}(F=8,27)$ :

$$
H_{C S}=\left(\begin{array}{cc}
4-8 \delta+\frac{26}{3} \delta^{2} & \frac{4 \sqrt{2}}{3} \delta+\frac{2 \sqrt{2}}{3} \delta^{2} \\
\frac{4 \sqrt{2}}{3} \delta+\frac{2 \sqrt{2}}{3} \delta^{2} & 24-\frac{80}{3} \delta+\frac{28}{3} \delta^{2}
\end{array}\right)
$$

\section{D. $q^{5} s^{4}:\{12345\}\{6789\}$}

(i) $I=\frac{5}{2}, S=\frac{3}{2}(F=64)$ :

$$
H_{C S}=56-\frac{128}{3} \delta+\frac{56}{3} \delta^{2}
$$

(ii) $I=\frac{5}{2}, S=\frac{1}{2}(F=\overline{35})$ :

$$
H_{C S}=40-\frac{80}{3} \delta+\frac{56}{3} \delta^{2}
$$

(iii) $I=\frac{3}{2}, S=\frac{5}{2}(F=27)$ :

$$
H_{C S}=\frac{104}{3}-\frac{112}{3} \delta+\frac{56}{3} \delta^{2}
$$

(iv) $I=\frac{3}{2}, S=\frac{3}{2}(F=\overline{10}, 27,64)$ :

$$
H_{C S}=\left(\begin{array}{ccc}
20-\frac{70}{3} \delta+\frac{170}{9} \delta^{2} & \frac{2}{3} \sqrt{\frac{35}{3}} \delta+\frac{2}{3} \sqrt{\frac{5}{21}} \delta^{2} & -\frac{4}{9} \sqrt{\frac{5}{7}} \delta^{2} \\
\frac{2}{3} \sqrt{\frac{35}{3}} \delta+\frac{2}{3} \sqrt{\frac{5}{21}} \delta^{2} & 28-\frac{674}{21} \delta+\frac{134}{7} \delta^{2} & -\frac{80}{21 \sqrt{3}} \delta-\frac{20}{21 \sqrt{3}} \delta^{2} \\
-\frac{4}{9} \sqrt{\frac{5}{7}} \delta^{2} & -\frac{80}{21 \sqrt{3}} \delta-\frac{20}{21 \sqrt{3}} \delta^{2} & 56-\frac{1216}{21} \delta+\frac{1216}{63} \delta^{2}
\end{array}\right)
$$

(v) $I=\frac{3}{2}, S=\frac{1}{2}(F=27,35, \overline{35})$ :

$$
H_{C S}=\left(\begin{array}{ccc}
24-\frac{86}{3} \delta+19 \delta^{2} & \frac{8}{3 \sqrt{3}} \delta+\frac{2}{3 \sqrt{3}} \delta^{2} & -\frac{2}{3} \sqrt{\frac{5}{3}} \delta+\frac{1}{3} \sqrt{\frac{5}{3}} \delta^{2} \\
\frac{8}{3 \sqrt{3}} \delta+\frac{2}{3 \sqrt{3}} \delta^{2} & 40-\frac{136}{3} \delta+\frac{172}{9} \delta^{2} & \frac{2 \sqrt{5}}{9} \delta^{2} \\
-\frac{2}{3} \sqrt{\frac{5}{3}} \delta+\frac{1}{3} \sqrt{\frac{5}{3}} \delta^{2} & \frac{2 \sqrt{5}}{9} \delta^{2} & 40-\frac{130}{3} \delta+\frac{173}{9} \delta^{2}
\end{array}\right)
$$

(vi) $I=\frac{1}{2}, S=\frac{7}{2}(F=8)$ :

$$
H_{C S}=24-32 \delta+\frac{56}{3} \delta^{2}
$$

(vii) $I=\frac{1}{2}, S=\frac{5}{2}(F=8,27)$ :

$$
H_{C S}=\left(\begin{array}{cc}
\frac{44}{3}-\frac{424}{15} \delta+\frac{56}{3} \delta^{2} & -\frac{8}{5} \sqrt{\frac{7}{3}} \delta \\
-\frac{8}{5} \sqrt{\frac{7}{3}} \delta & \frac{104}{3}-\frac{656}{15} \delta+\frac{56}{3} \delta^{2}
\end{array}\right)
$$


(viii) $I=\frac{1}{2}, S=\frac{3}{2}(F=8,10,27,64)$ :

$$
H_{C S}=\left(\begin{array}{cccc}
8-\frac{108}{5} \delta+\frac{58}{3} \delta^{2} & 2 \sqrt{\frac{2}{3}} \delta+\frac{1}{3} \sqrt{\frac{2}{3}} \delta^{2} & \frac{14 \sqrt{14}}{15} \delta+\frac{1}{3} \sqrt{\frac{2}{7}} \delta^{2} & \frac{8}{3 \sqrt{21}} \delta^{2} \\
2 \sqrt{\frac{2}{3}} \delta+\frac{1}{3} \sqrt{\frac{2}{3}} \delta^{2} & 20-\frac{106}{3} \delta+\frac{169}{9} \delta^{2} & -\frac{2}{3} \sqrt{\frac{7}{3}} \delta+\frac{1}{3 \sqrt{21}} \delta^{2} & \frac{4}{9} \sqrt{\frac{2}{7}} \delta^{2} \\
\frac{14 \sqrt{14}}{15} \delta+\frac{1}{3} \sqrt{\frac{2}{7}} \delta^{2} & -\frac{2}{3} \sqrt{\frac{7}{3}} \delta+\frac{1}{3 \sqrt{21}} \delta^{2} & 28-\frac{4342}{105} \delta+\frac{131}{7} \delta^{2} & -\frac{80}{21} \sqrt{\frac{2}{3}} \delta+\frac{4}{21} \sqrt{\frac{2}{3}} \delta^{2} \\
\frac{8}{3 \sqrt{21}} \delta^{2} & \frac{4}{9} \sqrt{\frac{2}{7}} \delta^{2} & -\frac{80}{21} \sqrt{\frac{2}{3}} \delta+\frac{4}{21} \sqrt{\frac{2}{3}} \delta^{2} & 56-\frac{1408}{21} \delta+\frac{1208}{63} \delta^{2}
\end{array}\right)
$$

(ix) $I=\frac{1}{2}, S=\frac{1}{2}(F=8,27,35)$ :

$$
H_{C S}=\left(\begin{array}{ccc}
4-\frac{64}{3} \delta+\frac{172}{9} \delta^{2} & -\frac{8}{3 \sqrt{3}} \delta-\frac{2}{3 \sqrt{3}} \delta^{2} & -\frac{2}{9} \sqrt{5} \delta^{2} \\
-\frac{8}{3 \sqrt{3}} \delta-\frac{2}{3 \sqrt{3}} \delta^{2} & 24-\frac{122}{3} \delta+19 \delta^{2} & -\frac{2}{3} \sqrt{\frac{5}{3}} \delta+\frac{1}{3} \sqrt{\frac{5}{3}} \delta^{2} \\
\frac{1}{9}(-2) \sqrt{5} \delta^{2} & -\frac{2}{3} \sqrt{\frac{5}{3}} \delta+\frac{1}{3} \sqrt{\frac{5}{3}} \delta^{2} & 40-\frac{166}{3} \delta+\frac{173}{9} \delta^{2}
\end{array}\right)
$$

\section{E. $q^{4} s^{5}:\{1234\}\{56789\}$}

(i) $I=2, S=\frac{3}{2}(F=64)$ :

$$
H_{C S}=56-\frac{208}{3} \delta+32 \delta^{2}
$$

(ii) $I=2, S=\frac{1}{2}(F=\overline{35})$ :

$$
H_{C S}=40-\frac{160}{3} \delta+32 \delta^{2}
$$

(iii) $I=1, S=\frac{5}{2}(F=27)$ :

$$
H_{C S}=\frac{104}{3}-\frac{176}{3} \delta+32 \delta^{2}
$$

(iv) $I=1, S=\frac{3}{2}(F=27,64)$ :

$$
H_{C S}=\left(\begin{array}{cc}
28-\frac{1172}{21} \delta+32 \delta^{2} & \frac{16 \sqrt{10}}{21} \delta \\
\frac{16 \sqrt{10}}{21} \delta & 56-\frac{1712}{21} \delta+32 \delta^{2}
\end{array}\right)
$$

(v) $I=1, S=\frac{1}{2}(F=27,35)$ :

$$
H_{C S}=\left(\begin{array}{cc}
24-\frac{164}{3} \delta+32 \delta^{2} & -\frac{4}{3} \delta \\
-\frac{4}{3} \delta & 40-\frac{212}{3} \delta+32 \delta^{2}
\end{array}\right)
$$

(vi) $I=0, S=\frac{3}{2}(F=10)$ :

$$
H_{C S}=20-\frac{172}{3} \delta+32 \delta^{2}
$$

(vii) $I=0, S=\frac{1}{2}(F=35)$ :

$$
H_{C S}=40-\frac{232}{3} \delta+32 \delta^{2}
$$

\section{F. $q^{3} s^{6}:\{123\}\{456789\}$}

(i) $I=\frac{3}{2}, S=\frac{3}{2}(F=64)$ :

$$
H_{C S}=56-96 \delta+48 \delta^{2}
$$

(ii) $I=\frac{1}{2}, S=\frac{1}{2}(F=35)$ :

$$
H_{C S}=40-96 \delta+48 \delta^{2}
$$

\section{STATIC BINDING ENERGY OF A TRIBARYON}

Now, we can calculate the binding energy of a tribaryon using the above matrix elements. Here, we represent the static binding energy of a tribaryon for the lowest flavor channels for each spin state using the color-spin matrix elements,

$$
\begin{gathered}
H=\frac{A}{m_{u} m_{u}} H_{C S}, \\
E_{B}=H^{\text {Tribaryon }}-H^{\text {Threshold }},
\end{gathered}
$$

where $A$ is fit to the proton-delta mass difference [21]. Additionally, we fix $\delta=\frac{2}{5}$ from taking $m_{u}=300 \mathrm{MeV}$ and $m_{s}=500 \mathrm{MeV}$. Here, $H^{\text {Threshold }}$ is the sum of the color spin interaction in Eq. (10) for the threshold, for which we consider only the three baryon states decaying into relative s-waves. 
TABLE I. The static binding energy of a tribaryon. The second row represents the most attractive flavor state of a tribaryon for the corresponding spin state. The third and fourth rows are the color-spin matrix elements of a tribaryon and the corresponding threshold both in the flavor $\mathrm{SU}(3)$ symmetric case. $E_{B}$ is the static binding energy of a tribaryon in the flavor $\mathrm{SU}(3)$ broken case in the MeV unit.

\begin{tabular}{|c|c|c|c|c|c|}
\hline & $S=\frac{9}{2}$ & $S=\frac{7}{2}$ & $S=\frac{5}{2}$ & $S=\frac{3}{2}$ & $S=\frac{1}{2}$ \\
\hline Flavor state & $F_{1}: q^{6} s^{3}(I=0)$ & $F_{8}: q^{7} s^{2}\left(I=\frac{1}{2}\right)$ & $F_{1}: q^{6} s^{3}(I=0)$ & $F_{1}: q^{6} s^{3}(I=0)$ & $F_{8}: q^{7} s^{2}\left(I=\frac{1}{2}\right)$ \\
\hline$H_{C S}^{\text {Tribaryon }}$ & 24 & 24 & $\frac{8}{3}$ & -4 & 4 \\
\hline$H_{C S}^{\text {Threshold }}$ & 24 & 24 & 8 & 8 & 8 \\
\hline Binding energy & $\begin{array}{c}\Delta \Xi^{*} \Sigma^{*} \\
E_{B}=17.07 \\
\Delta \Delta \Omega \\
E_{B}=0 \\
\Sigma^{*} \Sigma^{*} \Sigma^{*} \\
E_{B}=25.6\end{array}$ & $\begin{array}{c}\Delta \Delta \Xi^{*} \\
E_{B}=85.33 \\
\Delta \Sigma^{*} \Sigma^{*} \\
E_{B}=93.87\end{array}$ & $\begin{array}{c}\Delta \Xi^{*} \Sigma \\
E_{B}=-100.98 \\
\Delta \Xi \Sigma^{*} \\
E_{B}=-100.98 \\
N \Xi^{*} \Sigma^{*} \\
E_{B}=27.02 \\
\Lambda \Sigma^{*} \Sigma^{*} \\
E_{B}=-7.11\end{array}$ & $\begin{array}{c}\Delta \Xi^{*} \Sigma \\
E_{B}=-197.87 \\
\Delta \Xi \Sigma^{*} \\
E_{B}=-197.87 \\
N \Xi^{*} \Sigma^{*} \\
E_{B}=-69.87 \\
\Lambda \Sigma^{*} \Sigma^{*} \\
E_{B}=-104.0\end{array}$ & $\begin{array}{c}N \Delta \Xi^{*} \\
E_{B}=92.09 \\
N \Sigma^{*} \Sigma^{*} \\
E_{B}=100.62 \\
\Delta \Lambda \Sigma^{*} \\
E_{B}=57.96 \\
\Delta \Delta \Xi \\
E_{B}=-35.91 \\
\end{array}$ \\
\hline
\end{tabular}

For $S=\frac{9}{2}$, there is only one possible flavor state, which is the flavor singlet. In this case, $H_{C S}=24$ in the flavor $\mathrm{SU}(3)$ symmetric case. This state has three strange quarks and zero isospin, so that the possible lowest thresholds are $\Delta \Xi^{*} \Sigma^{*}, \Delta \Delta \Omega$, and $\Sigma^{*} \Sigma^{*} \Sigma^{*}$. For $S=\frac{7}{2}$, the flavor octet is the only unique flavor state. For this case, the possible number of strange quarks can be from 2 to 4 . Here, we only consider the tribaryon with two strange quarks for the flavor octet because the interaction usually becomes relatively repulsive as the number of quarks with the same flavor increases. For the flavor octet with two strange quarks and $S=\frac{7}{2}, H_{C S}=24$. In this case, the lowest threshold is two decuplet and one octet baryons. However, the sum of $H_{C S}$ for the lowest threshold is always lower than the corresponding tribaryon state, so we only consider possible resonances involving three decuplet baryons. Similarly, the lowest threshold is always more attractive for tribaryon configurations with $S=\frac{5}{2}, \frac{3}{2}, \frac{1}{2}$. Therefore, for these cases, we take the threshold to be those close to or higher than the tribaryon attraction to probe possible resonance states. We represent the static binding energy of a tribaryon for the lowest cases for each spin configurations in the flavor $\mathrm{SU}(3)$ broken case in Table I. As we can see in the table, the static binding energy is almost $-200 \mathrm{MeV}$ for some cases. However, it should be noted that for the tribaryon state to form a compact state the static binding energy has to be larger than the additional kinetic energy to form the compact configuration, which is a few hundred $\mathrm{MeV}$ for a typical hadron size state [21].

\section{DIQUARK CONFIGURATION OF A TRIBARYON}

In this section, we analyze the diquark structure of a tribaryon in the flavor SU(3) broken case. By analyzing the diquark structure of a multiparticle system, we can reconstruct the diagonal elements of the color-spin or flavor-spin interaction by adding the contribution of the corresponding interaction strength of all diquark configurations [10]. Additionally, the information of the detailed diquark configurations would also be useful to analyze the interaction originating from the meson exchange between quarks [2].

The way to calculate the diquark configuration is as follows. Let us examine a diquark component between $u, d$ quarks. Considering the symmetry, it is sufficient to calculate the diquark component between 1 and 2. First, for a corresponding flavor state, assemble the YoungYamanouchi basis into two sets that are symmetric and antisymmetric under the exchange between 1 and 2, respectively. Similarly, we can also collect the symmetric and antisymmetric basis set for color and spin states. Then, by taking the sum of the expectation value, we can calculate the diquark configuration for $u, d$ quarks as

$$
P_{12}(F, I, S)=\sum_{F, C, S}\left\langle\Psi_{F C S}\left|\hat{\mathcal{P}}_{12}\right| \Psi_{F C S}\right\rangle
$$

with a projection operator, $\hat{\mathcal{P}}_{i j} \equiv\left|\psi_{i j}^{\mathrm{d}}\right\rangle\left\langle\psi_{i j}^{\mathrm{d}}\right| \otimes \mathbf{1}$, where $\psi_{i j}^{\mathrm{d}}$ is the wave function of the relevant diquark that satisfies a certain symmetry property, $\mathbf{1}$ is a unit operator acting on the particles other than $i$ and $j$, and $\Psi_{F C S}$ is the wave function of a tribaryon.

For the diquark structure involving $s$ quarks, let us consider the $q^{6} s^{3}$ case again. In this case, because of the symmetric property, we only need to consider $P_{67}(F, I, S)$ and $P_{89}(F, I, S)$ for the diquark structure involving $u$ and $s$ quarks and $s$ quarks only, respectively. However, unless the particle label is 1 and 2, it is not easy to find the symmetric or antisymmetric basis set by just looking at the YoungYamanouchi basis. Still, there is a useful way to find the symmetric or antisymmetric basis set. The eigenvalues of 
TABLE II. The number of basis for symmetric and antisymmetric representation. For the conjugate Young diagram, the numbers of symmetric and antisymmetric basis are reversed.

\begin{tabular}{lccccccccccccccccc}
\hline \hline & {$[9]$} & {$[81]$} & {$[72]$} & {$[711]$} & {$[63]$} & {$[621]$} & {$[6111]$} & {$[54]$} & {$[531]$} & {$[522]$} & {$[5211]$} & {$[51111]$} & {$[441]$} & {$[432]$} & {$[4311]$} & {$[333]$} \\
\hline Symmetric & 1 & 7 & 21 & 21 & 34 & 70 & 35 & 28 & 99 & 70 & 105 & 35 & 49 & 91 & 111 & 21 \\
Antisymmetric & 0 & 1 & 6 & 7 & 14 & 35 & 21 & 14 & 63 & 50 & 84 & 35 & 35 & 77 & 105 & 21 \\
\hline \hline
\end{tabular}

TABLE III. The classification of two quark interaction. The two-quark state is determined to satisfy Pauli exclusion principle. We denote the antisymmetric and symmetric states as $A$ and $S$, respectively. The symbols inside the parentheses represent the multiplet state.

\begin{tabular}{lcccc}
\hline \hline \multicolumn{5}{c}{$q_{i} q_{j}$} \\
\hline Flavor & $A(\overline{3})$ & $S(6)$ & $A(\overline{3})$ & $S(6)$ \\
Color & $A(\overline{3})$ & $A(\overline{3})$ & $S(6)$ & $S(6)$ \\
Spin & $A(1)$ & $S(3)$ & $S(3)$ & $A(1)$ \\
$-\lambda_{i} \lambda_{j} \sigma_{i} \cdot \sigma_{j}$ & -8 & $\frac{8}{3}$ & $-\frac{4}{3}$ & 4 \\
\hline \hline
\end{tabular}

the elements $(i, j)$ of the irreducible representation of the corresponding Young diagram are 1 or -1 . Then, the eigenvectors with eigenvalue of 1 become the symmetric basis set, and the opposite case becomes the antisymmetric basis set. $\psi_{i j}^{d}$ in Eq. (12) is an element of these basis sets. Now, using Eq. (12), we can calculate the corresponding diquark component with a certain symmetric property. Additionally, the number of symmetric or antisymmetric basis between any $i$ and $j$ in a specific Young diagram is the same as the corresponding number between 1 and 2 . The numbers of symmetric and antisymmetric basis for $S_{9}$ Young diagrams are written in Table II.

Table III shows the basic building blocks composed of two quarks, henceforth called diquarks, and their corresponding strengths for the color-spin interaction. Treating the quarks as identical particles, the diquarks can be in one of the four possible configurations depending on the symmetry property under the exchange of the flavor, color, and spin states.

We represent the diquark configuration of a tribaryon for all possible states in Tables IV-XI. Table IV shows the result in the flavor SU(3) symmetric case. Additionally, it should be noted that $u$ and $s$ are treated as different flavors and hence do not have to satisfy the Pauli exclusion principle in the flavor SU(3) broken case. So, there are in total eight cases as shown in different columns to represent all possible diquark structure involving $u$ and $s$ quarks. By comparing each table with Table IV, we can analyze how the diquark components change when the flavor symmetry is broken.

The most attractive tribaryon state is related to configurations where there are large fraction of attractive diquark configurations. As an example, the way to find the most attractive channel is to identify configuration that has large contributions from the A-color (antisymmetric) and A-spin diquark state. For the flavor SU(3) symmetric case, as we can see in Table IV, the flavor singlet with $S=\frac{3}{2}$ and flavor octet with $S=\frac{1}{2}$ have the largest A-color and A-spin diquark state contribution. We can check that the flavor singlet state is the most attractive state, as can be seen by letting $\delta=0$ in the color-spin matrix elements given in Sec. IV for the flavor symmetric case. For flavor singlet

TABLE IV. The probability $P_{i j}$ of a tribaryon for $(i, j)$ diquark pairs with $S, A$, and $M$ representing symmetric $(S)$, antisymmetric $(A S)$, and mixed $(M)$ combinations in the flavor $\mathrm{SU}(3)$ symmetric case.

\begin{tabular}{lcccc}
\hline \hline$q^{9}$ & \multicolumn{4}{c}{$i, j=1-9$} \\
\hline Flavor & $A$ & $S$ & $A$ & $S$ \\
Color & $A$ & $A$ & $S$ & $S$ \\
Spin & $A$ & $S$ & $S$ & $A$ \\
$P_{i j}\left(F=1, S=\frac{3}{2}\right)$ & $\frac{7}{48}$ & $\frac{17}{48}$ & $\frac{17}{48}$ & $\frac{7}{48}$ \\
$P_{i j}\left(F=1, S=\frac{5}{2}\right)$ & $\frac{1}{9}$ & $\frac{7}{18}$ & $\frac{7}{18}$ & $\frac{1}{9}$ \\
$P_{i j}\left(F=1, S=\frac{9}{2}\right)$ & 0 & $\frac{1}{2}$ & $\frac{1}{2}$ & 0 \\
$P_{i j}\left(F=8, S=\frac{1}{2}\right)$ & $\frac{7}{48}$ & $\frac{17}{48}$ & $\frac{5}{16}$ & $\frac{3}{16}$ \\
$P_{i j}\left(F=8, S=\frac{3}{2}\right)$ & $\frac{1}{8}$ & $\frac{3}{8}$ & $\frac{1}{3}$ & $\frac{1}{6}$ \\
$P_{i j}\left(F=8, S=\frac{5}{2}\right)$ & $\frac{13}{144}$ & $\frac{59}{144}$ & $\frac{53}{144}$ & $\frac{19}{144}$ \\
$P_{i j}\left(F=8, S=\frac{7}{2}\right)$ & $\frac{1}{24}$ & $\frac{11}{24}$ & $\frac{5}{12}$ & $\frac{1}{12}$ \\
$P_{i j}\left(F=10, S=\frac{3}{2}\right)$ & $\frac{5}{48}$ & $\frac{19}{48}$ & $\frac{5}{16}$ & $\frac{3}{16}$ \\
$P_{i j}\left(F=\overline{10}, S=\frac{3}{2}\right)$ & $\frac{5}{48}$ & $\frac{19}{48}$ & $\frac{5}{16}$ & $\frac{3}{16}$ \\
$P_{i j}\left(F=27, S=\frac{1}{2}\right)$ & $\frac{1}{9}$ & $\frac{7}{18}$ & $\frac{5}{18}$ & $\frac{2}{9}$ \\
$P_{i j}\left(F=27, S=\frac{3}{2}\right)$ & $\frac{13}{144}$ & $\frac{59}{144}$ & $\frac{43}{144}$ & $\frac{29}{144}$ \\
$P_{i j}\left(F=27, S=\frac{5}{2}\right)$ & $\frac{1}{18}$ & $\frac{4}{9}$ & $\frac{1}{3}$ & $\frac{1}{6}$ \\
$P_{i j}\left(F=35, S=\frac{1}{2}\right)$ & $\frac{1}{12}$ & $\frac{5}{12}$ & $\frac{1}{4}$ & $\frac{1}{4}$ \\
$P_{i j}\left(F=\overline{35}, S=\frac{1}{2}\right)$ & $\frac{1}{12}$ & $\frac{5}{12}$ & $\frac{1}{4}$ & $\frac{1}{4}$ \\
$P_{i j}\left(F=64, S=\frac{3}{2}\right)$ & $\frac{1}{24}$ & $\frac{11}{24}$ & $\frac{1}{4}$ & $\frac{1}{4}$ \\
\hline \hline
\end{tabular}

TABLE V. The probability $P_{i j}$ of $q^{9}$ for $(i, j)$ diquark pairs with $S, A$, and $M$ representing symmetric $(S)$, antisymmetric $(A S)$, and mixed $(M)$ combinations in the flavor $\mathrm{SU}(3)$ broken case.

\begin{tabular}{lcccc}
\hline \hline$q^{9}$ & \multicolumn{4}{c}{$i, j=1-9$} \\
\hline Flavor & $A$ & $S$ & $A$ & $S$ \\
Color & $A$ & $A$ & $S$ & $S$ \\
Spin & $A$ & $S$ & $S$ & $A$ \\
$P_{i j}\left(F=\overline{35}, I=\frac{1}{2}, S=\frac{1}{2}\right)$ & $\frac{1}{12}$ & $\frac{5}{12}$ & $\frac{1}{4}$ & $\frac{1}{4}$ \\
$P_{i j}\left(F=64, I=\frac{3}{2}, S=\frac{3}{2}\right)$ & $\frac{1}{24}$ & $\frac{11}{24}$ & $\frac{1}{4}$ & $\frac{1}{4}$ \\
\hline \hline
\end{tabular}


TABLE VI. Same as Table V for $q^{8} s$.

\begin{tabular}{|c|c|c|c|c|c|c|c|c|c|c|c|c|}
\hline \multirow{2}{*}{$\frac{q^{8} s}{\text { Flavor }}$} & \multicolumn{4}{|c|}{$i, j=1-8$} & \multicolumn{8}{|c|}{$i=1-8, j=9$} \\
\hline & $A$ & $S$ & $A$ & $S$ & $A$ & $S$ & $A$ & $S$ & $A$ & $S$ & $A$ & $S$ \\
\hline Color & $A$ & $A$ & $S$ & $S$ & $A$ & $A$ & $S$ & $S$ & $S$ & $S$ & $A$ & $A$ \\
\hline Spin & $A$ & $S$ & $S$ & $A$ & $A$ & $S$ & $S$ & $A$ & $A$ & $S$ & $S$ & $A$ \\
\hline$P_{i j}\left(F=64, I=2, S=\frac{3}{2}\right)$ & $\frac{1}{28}$ & $\frac{13}{28}$ & $\frac{3}{14}$ & $\frac{2}{7}$ & $\frac{5}{128}$ & $\frac{45}{128}$ & $\frac{15}{64}$ & $\frac{3}{64}$ & $\frac{5}{64}$ & $\frac{9}{64}$ & $\frac{11}{128}$ & $\frac{3}{128}$ \\
\hline$P_{i j}\left(F=35, I=2, S=\frac{1}{2}\right)$ & $\frac{1}{28}$ & $\frac{13}{28}$ & $\frac{3}{14}$ & $\frac{2}{7}$ & $\frac{41}{224}$ & $\frac{3}{32}$ & $\frac{3}{14}$ & $\frac{3}{56}$ & $\frac{1}{14}$ & $\frac{9}{56}$ & $\frac{5}{32}$ & $\frac{15}{224}$ \\
\hline$P_{i j}\left(F=27, I=1, S=\frac{5}{2}\right)$ & $\frac{1}{28}$ & $\frac{13}{28}$ & $\frac{2}{7}$ & $\frac{3}{14}$ & $\frac{7}{96}$ & $\frac{41}{288}$ & $\frac{23}{72}$ & 0 & 0 & $\frac{13}{72}$ & $\frac{67}{288}$ & $\frac{5}{96}$ \\
\hline$P_{i j}\left(F=64, I=1, S=\frac{3}{2}\right)$ & $\frac{9}{196}$ & $\frac{89}{196}$ & $\frac{27}{98}$ & $\frac{11}{49}$ & $\frac{9}{896}$ & $\frac{369}{896}$ & $\frac{27}{448}$ & $\frac{127}{448}$ & $\frac{25}{448}$ & $\frac{45}{448}$ & $\frac{55}{896}$ & $\frac{15}{896}$ \\
\hline$P_{i j}\left(F=27, I=1, S=\frac{3}{2}\right)$ & $\frac{3}{49}$ & $\frac{43}{98}$ & $\frac{51}{196}$ & $\frac{47}{196}$ & $\frac{983}{8064}$ & $\frac{107}{896}$ & $\frac{727}{2688}$ & $\frac{185}{8064}$ & $\frac{355}{8064}$ & $\frac{437}{2688}$ & $\frac{169}{896}$ & $\frac{565}{8064}$ \\
\hline$P_{i j}\left(F=\overline{35}, I=1, S=\frac{1}{2}\right)$ & $\frac{9}{112}$ & $\frac{47}{112}$ & $\frac{27}{112}$ & $\frac{29}{112}$ & $\frac{9}{128}$ & $\frac{45}{128}$ & $\frac{27}{128}$ & $\frac{23}{128}$ & $\frac{5}{128}$ & $\frac{9}{128}$ & $\frac{7}{128}$ & $\frac{3}{128}$ \\
\hline$P_{i j}\left(F=27, I=1, S=\frac{1}{2}\right)$ & $\frac{9}{112}$ & $\frac{47}{112}$ & $\frac{27}{112}$ & $\frac{29}{112}$ & $\frac{167}{1152}$ & $\frac{41}{384}$ & $\frac{95}{384}$ & $\frac{41}{1152}$ & $\frac{67}{1152}$ & $\frac{61}{384}$ & $\frac{67}{384}$ & $\frac{85}{1152}$ \\
\hline$P_{i j}\left(F=\overline{10}, I=0, S=\frac{3}{2}\right)$ & $\frac{5}{56}$ & $\frac{23}{56}$ & $\frac{15}{56}$ & $\frac{13}{56}$ & $\frac{25}{256}$ & $\frac{33}{256}$ & $\frac{75}{256}$ & $\frac{3}{256}$ & $\frac{5}{256}$ & $\frac{45}{256}$ & $\frac{55}{256}$ & $\frac{15}{256}$ \\
\hline$P_{i j}\left(F=\overline{35}, I=0, S=\frac{1}{2}\right)$ & $\frac{5}{56}$ & $\frac{23}{56}$ & $\frac{15}{56}$ & $\frac{13}{56}$ & $\frac{1}{64}$ & $\frac{21}{64}$ & $\frac{3}{64}$ & $\frac{15}{64}$ & $\frac{5}{64}$ & $\frac{9}{64}$ & $\frac{7}{64}$ & $\frac{3}{64}$ \\
\hline
\end{tabular}

with $S=\frac{3}{2}, H_{C S}$ is -4 , and for flavor octet with $S=\frac{1}{2}, H_{C S}$ is 4. This difference comes from the remaining diquark components other than A-color and A-spin states. As can be seen in Table III, there are two attractive diquarks. The reason why flavor singlet state is more attractive is that, compared to the flavor octet case, it has larger contributions from the other remaining attractive diquark components, which is the S-color(symmetric) and $\mathrm{S}$-spin state. Therefore, the flavor states which have large components of A-color, A-spin, and S-color, S-spin states lead to attractive configurations. For the flavor symmetry broken case, it is still useful to search for the diquark components between $u$ and $d$ quarks to find the most attractive channel. The following are the most attractive diagonal channels for all possible strangeness quantum numbers, up to six strange quarks, in the flavor $\mathrm{SU}(3)$ broken case:

(i) $q^{9}:(F, I, S)=\left(\overline{35}, \frac{1}{2}, \frac{1}{2}\right)$

(ii) $q^{8} s:(F, I, S)=\left(\overline{10}, 0, \frac{3}{2}\right),\left(\overline{35}, 0, \frac{1}{2}\right)$

(iii) $q^{7} s^{2}:(F, I, S)=\left(27, \frac{1}{2}, \frac{1}{2}\right)$

(iv) $q^{6} s^{3}:(F, I, S)=\left(27,0, \frac{1}{2}\right)$

(v) $q^{5} s^{4}:(F, I, S)=\left(8, \frac{1}{2}, \frac{1}{2}\right)$

(vi) $q^{4} s^{5}:(F, I, S)=\left(10,0, \frac{3}{2}\right),\left(35,0, \frac{1}{2}\right)$

(vii) $q^{3} s^{6}:(F, I, S)=\left(35, \frac{1}{2}, \frac{1}{2}\right)$

To obtained the lowest eigenvalue for the present case, we need to consider the mixing effects between different $\mathrm{SU}(3)$ flavor channels and diagonalize the whole matrix components in Sec. IV.

\section{CONCLUSIONS}

In this work, we calculate the matrix elements of colorspin interaction of a tribaryon in the flavor $\mathrm{SU}(3)$ broken case. To calculate this, we construct the flavor $\otimes$ color $\otimes$ spin wave function of a tribaryon to satisfy the Pauli principle. Additionally, we analyze the diquark structure of a tribaryon by using the symmetric and antisymmetric basis set of each Young tableau of $S_{9}$ symmetric group. By looking at the largest diquark components for antisymmetric color and spin states, we can find the most attractive channels of a tribaryon. Furthermore, The diquark structure table can be useful in analyzing the flavor-spin interactions as well as the color-spin interactions of a tribaryon.

\section{ACKNOWLEDGMENTS}

The work by S. H. L. was supported by Samsung Science and Technology Foundation under Project Number SSTFBA1901-04. This work by A.P. was supported by the Korea National Research Foundation under Grant No. 2018R1D1A1B07043234.

\section{APPENDIX: ORBITAL STATE OF A TRIBARYON}

In this section, we present the possible orbital state of a tribaryon, considering it as a combined state of three s-wave baryons. For a baryon, we can represent its orbital state using the Young diagram [3] since the orbital part is totally symmetric. It is also well known that there are in total four possible orbital states in a two-baryon system as follows:

$$
[3] \times[3]=[6]+[51]+[42]+[33] .
$$

By further applying the outer product of an orbital state of a baryon, we can get the following possible orbital state of a tribaryon,

$$
\begin{aligned}
{[3] \times } & {[3] \times[3] } \\
= & {[6] \times[3]+[51] \times[3]+[42] \times[3]+[33] \times[3] } \\
= & {[9]+[81]_{(m=2)}+[72]_{(m=3)}+[63]_{(m=4)}+[54]_{(m=2)} } \\
& +[711]+[621]_{(m=2)}+[531]_{(m=3)} \\
& +[522]+[432]_{(m=2)}+[333],
\end{aligned}
$$


TABLE VII. Same as Table V for $q^{7} s^{2}$.

\begin{tabular}{|c|c|c|c|c|c|c|c|c|c|c|c|c|c|c|}
\hline \multirow{2}{*}{$\frac{q^{7} s^{2}}{\text { Flavor }}$} & \multicolumn{4}{|c|}{$i, j=1-7$} & \multicolumn{2}{|c|}{$i, j=8,9$} & \multicolumn{8}{|c|}{$i=1-7, j=8,9$} \\
\hline & $A$ & $S$ & $A$ & $S$ & $S$ & $S$ & $A$ & $S$ & $A$ & $S$ & $A$ & $S$ & $A$ & $S$ \\
\hline Color & $A$ & $A$ & $S$ & $S$ & $S$ & $A$ & A & $A$ & $S$ & $S$ & $S$ & $S$ & A & $A$ \\
\hline Spin & $A$ & $S$ & $S$ & $A$ & $A$ & $S$ & A & $S$ & $S$ & $A$ & $A$ & $S$ & $S$ & $A$ \\
\hline$P_{i j}\left(F=64, I=\frac{5}{2}, S=\frac{3}{2}\right)$ & $\frac{1}{42}$ & $\frac{1}{2}$ & $\frac{1}{7}$ & $\frac{1}{3}$ & 1 & 0 & $\frac{3}{70}$ & $\frac{17}{70}$ & $\frac{9}{35}$ & $\frac{2}{35}$ & $\frac{3}{35}$ & $\frac{6}{35}$ & $\frac{4}{35}$ & $\frac{1}{35}$ \\
\hline$P_{i j}\left(F=35, I=\frac{5}{2}, S=\frac{1}{2}\right)$ & $\frac{1}{42}$ & $\frac{1}{2}$ & $\frac{1}{7}$ & $\frac{1}{3}$ & 1 & 0 & $\frac{489}{3920}$ & $\frac{11}{112}$ & $\frac{243}{980}$ & $\frac{59}{980}$ & $\frac{81}{980}$ & $\frac{177}{980}$ & $\frac{17}{112}$ & $\frac{211}{3920}$ \\
\hline$P_{i j}\left(F=27, I=\frac{3}{2}, S=\frac{5}{2}\right)$ & $\frac{1}{21}$ & $\frac{10}{21}$ & $\frac{5}{21}$ & $\frac{5}{21}$ & 1 & 0 & $\frac{11}{252}$ & $\frac{10}{63}$ & $\frac{2}{7}$ & $\frac{1}{36}$ & $\frac{11}{252}$ & $\frac{3}{14}$ & $\frac{25}{126}$ & $\frac{1}{36}$ \\
\hline$P_{i j}\left(F=10, I=\frac{3}{2}, S=\frac{3}{2}\right)$ & $\frac{3}{56}$ & $\frac{233}{504}$ & $\frac{13}{56}$ & $\frac{127}{504}$ & $\frac{5}{6}$ & $\frac{1}{6}$ & $\frac{59}{448}$ & $\frac{395}{4032}$ & $\frac{129}{448}$ & $\frac{145}{4032}$ & $\frac{227}{4032}$ & $\frac{75}{448}$ & $\frac{673}{4032}$ & $\frac{25}{448}$ \\
\hline$P_{i j}\left(F=27, I=\frac{3}{2}, S=\frac{3}{2}\right)$ & $\frac{71}{1176}$ & $\frac{25}{56}$ & $\frac{265}{1176}$ & $\frac{15}{56}$ & $\frac{9}{14}$ & $\frac{5}{14}$ & $\frac{1121}{14112}$ & $\frac{305}{2016}$ & $\frac{3527}{14112}$ & $\frac{71}{2016}$ & $\frac{781}{14112}$ & $\frac{2539}{14112}$ & $\frac{2635}{14112}$ & $\frac{877}{14112}$ \\
\hline$P_{i j}\left(F=64, I=\frac{3}{2}, S=\frac{3}{2}\right)$ & $\frac{2}{49}$ & $\frac{29}{63}$ & $\frac{12}{49}$ & $\frac{16}{63}$ & $\frac{11}{21}$ & $\frac{10}{21}$ & $\frac{67}{3920}$ & $\frac{1621}{5040}$ & $\frac{201}{1960}$ & $\frac{391}{2520}$ & $\frac{1283}{17640}$ & $\frac{339}{1960}$ & $\frac{4553}{35280}$ & $\frac{113}{3920}$ \\
\hline$P_{i j}\left(F=27, I=\frac{3}{2}, S=\frac{1}{2}\right)$ & $\frac{11}{168}$ & $\frac{25}{56}$ & $\frac{37}{168}$ & $\frac{15}{56}$ & $\frac{3}{4}$ & $\frac{1}{4}$ & $\frac{103}{1008}$ & $\frac{121}{1008}$ & $\frac{229}{1008}$ & $\frac{67}{1008}$ & $\frac{43}{504}$ & $\frac{79}{504}$ & $\frac{79}{504}$ & $\frac{43}{504}$ \\
\hline$P_{i j}\left(F=35, I=\frac{3}{2}, S=\frac{1}{2}\right)$ & $\frac{3}{56}$ & $\frac{227}{504}$ & $\frac{13}{56}$ & $\frac{19}{72}$ & $\frac{7}{12}$ & $\frac{5}{12}$ & $\frac{43}{560}$ & $\frac{53}{252}$ & $\frac{69}{560}$ & $\frac{167}{1260}$ & $\frac{61}{720}$ & $\frac{6}{35}$ & $\frac{145}{1008}$ & $\frac{2}{35}$ \\
\hline$P_{i j}\left(F=\overline{35}, I=\frac{3}{2}, S=\frac{1}{2}\right)$ & $\frac{1}{14}$ & $\frac{55}{126}$ & $\frac{3}{14}$ & $\frac{5}{18}$ & $\frac{2}{3}$ & $\frac{1}{3}$ & $\frac{1}{14}$ & $\frac{71}{252}$ & $\frac{3}{14}$ & $\frac{37}{252}$ & $\frac{1}{18}$ & $\frac{3}{28}$ & $\frac{11}{126}$ & $\frac{1}{28}$ \\
\hline$P_{i j}\left(F=8, I=\frac{1}{2}, S=\frac{7}{2}\right)$ & $\frac{1}{42}$ & $\frac{1}{2}$ & $\frac{1}{3}$ & $\frac{1}{7}$ & 1 & 0 & $\frac{3}{70}$ & $\frac{9}{70}$ & $\frac{13}{35}$ & 0 & 0 & $\frac{1}{5}$ & $\frac{8}{35}$ & $\frac{1}{35}$ \\
\hline$P_{i j}\left(F=27, I=\frac{1}{2}, S=\frac{5}{2}\right)$ & $\frac{1}{30}$ & $\frac{103}{210}$ & $\frac{34}{105}$ & $\frac{16}{105}$ & 1 & 0 & $\frac{199}{5040}$ & $\frac{4651}{25200}$ & $\frac{11}{60}$ & $\frac{691}{6300}$ & $\frac{569}{6300}$ & $\frac{79}{420}$ & $\frac{3809}{25200}$ & $\frac{269}{5040}$ \\
\hline$P_{i j}\left(F=8, I=\frac{1}{2}, S=\frac{5}{2}\right)$ & $\frac{31}{420}$ & $\frac{9}{20}$ & $\frac{17}{60}$ & $\frac{27}{140}$ & 1 & 0 & $\frac{9}{112}$ & $\frac{311}{2800}$ & $\frac{187}{560}$ & $\frac{7}{400}$ & $\frac{13}{400}$ & $\frac{3}{16}$ & $\frac{5499}{2800}$ & $\frac{23}{560}$ \\
\hline$P_{i j}\left(F=8, I=\frac{1}{2}, S=\frac{3}{2}\right)$ & $\frac{31}{420}$ & $\frac{179}{420}$ & $\frac{17}{60}$ & $\frac{13}{60}$ & $\frac{1}{2}$ & $\frac{1}{2}$ & $\frac{15}{112}$ & $\frac{883}{8400}$ & $\frac{157}{560}$ & $\frac{197}{8400}$ & $\frac{373}{8400}$ & $\frac{17}{112}$ & $\frac{221}{1200}$ & $\frac{43}{560}$ \\
\hline$P_{i j}\left(F=\overline{10}, I=\frac{1}{2}, S=\frac{3}{2}\right)$ & $\frac{5}{56}$ & $\frac{31}{72}$ & $\frac{15}{56}$ & $\frac{107}{504}$ & $\frac{11}{12}$ & $\frac{1}{12}$ & $\frac{5}{64}$ & $\frac{611}{4032}$ & $\frac{15}{64}$ & $\frac{361}{4032}$ & $\frac{275}{4032}$ & $\frac{75}{448}$ & $\frac{625}{4032}$ & $\frac{25}{448}$ \\
\hline$P_{i j}\left(F=27, I=\frac{1}{2}, S=\frac{3}{2}\right)$ & $\frac{421}{5880}$ & $\frac{883}{1960}$ & $\frac{1679}{5880}$ & $\frac{377}{1960}$ & $\frac{27}{28}$ & $\frac{1}{28}$ & $\frac{8507}{141120}$ & $\frac{122503}{705600}$ & $\frac{22937}{141120}$ & $\frac{85117}{705600}$ & $\frac{74903}{705600}$ & $\frac{24979}{141120}$ & $\frac{95477}{705600}$ & $\frac{9907}{141120}$ \\
\hline$P_{i j}\left(F=64, I=\frac{1}{2}, S=\frac{3}{2}\right)$ & $\frac{5}{98}$ & $\frac{401}{882}$ & $\frac{15}{49}$ & $\frac{83}{441}$ & $\frac{13}{21}$ & $\frac{8}{21}$ & $\frac{1}{98}$ & $\frac{331}{882}$ & $\frac{3}{49}$ & $\frac{118}{441}$ & $\frac{29}{441}$ & $\frac{6}{49}$ & $\frac{34}{441}$ & $\frac{1}{49}$ \\
\hline$P_{i j}\left(F=8, I=\frac{1}{2}, S=\frac{1}{2}\right)$ & $\frac{3}{28}$ & $\frac{101}{252}$ & $\frac{1}{4}$ & $\frac{61}{252}$ & $\frac{2}{3}$ & $\frac{1}{3}$ & $\frac{79}{560}$ & $\frac{481}{5040}$ & $\frac{153}{560}$ & $\frac{167}{5040}$ & $\frac{313}{5040}$ & $\frac{87}{560}$ & $\frac{839}{5040}$ & $\frac{41}{560}$ \\
\hline$P_{i j}\left(F=27, I=\frac{1}{2}, S=\frac{1}{2}\right)$ & $\frac{17}{168}$ & $\frac{23}{56}$ & $\frac{43}{168}$ & $\frac{13}{56}$ & $\frac{3}{4}$ & $\frac{1}{4}$ & $\frac{1409}{20160}$ & $\frac{3641}{20160}$ & $\frac{3083}{20160}$ & $\frac{2291}{20160}$ & $\frac{1849}{20160}$ & $\frac{511}{2880}$ & $\frac{3019}{20160}$ & $\frac{1291}{20160}$ \\
\hline$P_{i j}\left(F=\overline{35}, I=\frac{1}{2}, S=\frac{1}{2}\right)$ & $\frac{5}{56}$ & $\frac{209}{504}$ & $\frac{15}{56}$ & $\frac{115}{504}$ & $\frac{7}{12}$ & $\frac{5}{12}$ & $\frac{23}{448}$ & $\frac{1367}{4032}$ & $\frac{69}{448}$ & $\frac{901}{4032}$ & $\frac{191}{4032}$ & $\frac{39}{448}$ & $\frac{277}{4032}$ & $\frac{13}{448}$ \\
\hline
\end{tabular}

TABLE VIII. Same as Table V for $q^{6} s^{3}$.

\begin{tabular}{|c|c|c|c|c|c|c|c|c|c|c|c|c|c|c|}
\hline \multirow{2}{*}{$\frac{q^{6} s^{3}}{\text { Flavor }}$} & \multicolumn{4}{|c|}{$i, j=1-6$} & \multicolumn{2}{|c|}{$i, j=7-9$} & \multicolumn{8}{|c|}{$i=1-6, j=7-9$} \\
\hline & $A$ & $S$ & $A$ & $S$ & $S$ & $S$ & $A$ & $S$ & $A$ & $S$ & $A$ & $S$ & $A$ & $S$ \\
\hline Color & $A$ & $A$ & $S$ & $S$ & $S$ & $A$ & $A$ & $A$ & $S$ & $S$ & $S$ & $S$ & $A$ & $A$ \\
\hline Spin & $A$ & $S$ & $S$ & $A$ & $A$ & $S$ & $A$ & $S$ & $S$ & $A$ & $A$ & $S$ & $S$ & $A$ \\
\hline$P_{i j}\left(F=64, I=3, S=\frac{3}{2}\right)$ & 0 & $\frac{3}{5}$ & 0 & $\frac{2}{5}$ & 1 & 0 & $\frac{7}{144}$ & $\frac{5}{48}$ & $\frac{7}{24}$ & $\frac{5}{72}$ & $\frac{7}{72}$ & $\frac{5}{24}$ & $\frac{7}{48}$ & $\frac{5}{144}$ \\
\hline$P_{i j}\left(F=27, I=2, S=\frac{5}{2}\right)$ & $\frac{1}{15}$ & $\frac{8}{15}$ & $\frac{2}{15}$ & $\frac{4}{15}$ & 1 & 0 & $\frac{43}{1296}$ & $\frac{139}{1296}$ & $\frac{221}{648}$ & $\frac{29}{648}$ & $\frac{43}{648}$ & $\frac{139}{648}$ & $\frac{221}{1296}$ & $\frac{29}{1296}$ \\
\hline$P_{i j}\left(F=27, I=2, S=\frac{3}{2}\right)$ & $\frac{23}{420}$ & $\frac{73}{140}$ & $\frac{61}{420}$ & $\frac{39}{140}$ & $\frac{37}{42}$ & $\frac{5}{42}$ & $\frac{761}{9072}$ & $\frac{281}{3024}$ & $\frac{439}{1512}$ & $\frac{67}{1134}$ & $\frac{52}{567}$ & $\frac{281}{1512}$ & $\frac{439}{3024}$ & $\frac{463}{9072}$ \\
\hline$P_{i j}\left(F=64, I=2, S=\frac{3}{2}\right)$ & $\frac{1}{53}$ & $\frac{52}{105}$ & $\frac{6}{35}$ & $\frac{32}{105}$ & $\frac{13}{21}$ & $\frac{8}{21}$ & $\frac{29}{1008}$ & $\frac{29}{112}$ & $\frac{29}{168}$ & $\frac{55}{504}$ & $\frac{37}{504}$ & $\frac{31}{168}$ & $\frac{143}{1008}$ & $\frac{31}{1008}$ \\
\hline$P_{i j}\left(F=27, I=2, S=\frac{1}{2}\right)$ & $\frac{1}{30}$ & $\frac{1}{2}$ & $\frac{1}{6}$ & $\frac{3}{10}$ & $\frac{2}{3}$ & $\frac{1}{3}$ & $\frac{79}{648}$ & $\frac{7}{72}$ & $\frac{109}{432}$ & $\frac{71}{1296}$ & $\frac{109}{1296}$ & $\frac{71}{432}$ & $\frac{11}{72}$ & $\frac{47}{648}$ \\
\hline$P_{i j}\left(F=35, I=2, S=\frac{1}{2}\right)$ & $\frac{1}{20}$ & $\frac{29}{60}$ & $\frac{3}{20}$ & $\frac{19}{60}$ & $\frac{2}{3}$ & $\frac{1}{3}$ & $\frac{43}{576}$ & $\frac{281}{1728}$ & $\frac{35}{192}$ & $\frac{163}{1728}$ & $\frac{149}{1728}$ & $\frac{37}{192}$ & $\frac{271}{1728}$ & $\frac{29}{576}$ \\
\hline$P_{i j}\left(F=\overline{35}, I=2, S=\frac{1}{2}\right)$ & $\frac{1}{20}$ & $\frac{29}{60}$ & $\frac{3}{20}$ & $\frac{19}{60}$ & $\frac{2}{3}$ & $\frac{1}{3}$ & $\frac{5}{64}$ & $\frac{13}{64}$ & $\frac{15}{64}$ & $\frac{7}{64}$ & $\frac{41}{576}$ & $\frac{9}{64}$ & $\frac{67}{5768}$ & $\frac{3}{64}$ \\
\hline$P_{i j}\left(F=8, I=1, S=\frac{7}{2}\right)$ & $\frac{1}{15}$ & $\frac{8}{15}$ & $\frac{4}{15}$ & $\frac{2}{15}$ & 1 & 0 & $\frac{37}{2160}$ & $\frac{79}{720}$ & $\frac{47}{120}$ & $\frac{23}{1080}$ & $\frac{37}{1080}$ & $\frac{79}{360}$ & $\frac{47}{240}$ & $\frac{23}{2160}$ \\
\hline$P_{i j}\left(F=8, I=1, S=\frac{5}{2}\right)$ & $\frac{13}{300}$ & $\frac{51}{100}$ & $\frac{29}{100}$ & $\frac{47}{300}$ & $\frac{11}{15}$ & $\frac{4}{15}$ & $\frac{251}{2700}$ & $\frac{173}{1800}$ & $\frac{379}{1200}$ & $\frac{377}{10800}$ & $\frac{643}{10800}$ & $\frac{643}{3600}$ & $\frac{307}{1800}$ & $\frac{139}{2700}$ \\
\hline$P_{i j}\left(F=27, I=1, S=\frac{5}{2}\right)$ & $\frac{1}{25}$ & $\frac{38}{75}$ & $\frac{22}{75}$ & $\frac{4}{25}$ & $\frac{11}{15}$ & $\frac{4}{15}$ & $\frac{421}{10800}$ & $\frac{1877}{10800}$ & $\frac{1147}{5400}$ & $\frac{419}{5400}$ & $\frac{421}{5400}$ & $\frac{1133}{5400}$ & $\frac{1843}{10800}$ & $\frac{419}{10800}$ \\
\hline$P_{i j}\left(F=8, I=1, S=\frac{3}{2}\right)$ & $\frac{19}{150}$ & $\frac{197}{450}$ & $\frac{31}{150}$ & $\frac{103}{450}$ & $\frac{37}{45}$ & $\frac{8}{45}$ & $\frac{251}{2700}$ & $\frac{121}{1350}$ & $\frac{379}{1200}$ & $\frac{149}{3600}$ & $\frac{773}{10800}$ & $\frac{643}{3600}$ & $\frac{107}{675}$ & $\frac{139}{2700}$ \\
\hline$P_{i j}\left(F=10, I=1, S=\frac{3}{2}\right)$ & $\frac{1}{12}$ & $\frac{25}{54}$ & $\frac{1}{4}$ & $\frac{11}{54}$ & $\frac{79}{108}$ & $\frac{29}{108}$ & $\frac{23}{270}$ & $\frac{209}{1620}$ & $\frac{7}{30}$ & $\frac{127}{1620}$ & $\frac{133}{1620}$ & $\frac{11}{60}$ & $\frac{251}{1620}$ & $\frac{29}{540}$ \\
\hline$P_{i j}\left(F=\overline{10}, I=1, S=\frac{3}{2}\right)$ & $\frac{1}{12}$ & $\frac{25}{54}$ & $\frac{1}{4}$ & $\frac{11}{54}$ & $\frac{79}{108}$ & $\frac{29}{108}$ & $\frac{35}{432}$ & $\frac{173}{1296}$ & $\frac{35}{144}$ & $\frac{103}{1296}$ & $\frac{35}{432}$ & $\frac{25}{144}$ & $\frac{65}{432}$ & $\frac{25}{432}$ \\
\hline
\end{tabular}


TABLE VIII. (Continued)

\begin{tabular}{|c|c|c|c|c|c|c|c|c|c|c|c|c|c|c|}
\hline \multicolumn{2}{|l|}{$q^{6} s^{3}$} & \multicolumn{3}{|c|}{$i, j=1-6$} & \multicolumn{2}{|c|}{$i, j=7-9$} & \multicolumn{8}{|c|}{$i=1-6, j=7-9$} \\
\hline$P_{i j}\left(F=27, I=1, S=\frac{3}{2}\right)$ & $\frac{53}{700}$ & $\frac{949}{2100}$ & $\frac{541}{2100}$ & $\frac{451}{2100}$ & $\frac{67}{105}$ & $\frac{38}{105}$ & $\frac{4397}{75600}$ & $\frac{12829}{75600}$ & $\frac{913}{4725}$ & $\frac{386}{4725}$ & $\frac{773}{9450}$ & $\frac{1789}{9450}$ & $\frac{12611}{75600}$ & $\frac{4483}{75600}$ \\
\hline$P_{i j}\left(F=64, I=1, S=\frac{3}{2}\right)$ & $\frac{1}{21}$ & $\frac{442}{945}$ & $\frac{2}{7}$ & $\frac{188}{945}$ & $\frac{109}{189}$ & $\frac{80}{189}$ & $\frac{47}{3024}$ & $\frac{935}{3024}$ & $\frac{47}{504}$ & $\frac{95}{504}$ & $\frac{341}{4536}$ & $\frac{85}{504}$ & $\frac{1103}{9072}$ & $\frac{85}{3024}$ \\
\hline$P_{i j}\left(F=8, I=1, S=\frac{1}{2}\right)$ & $\frac{1}{12}$ & $\frac{241}{540}$ & $\frac{1}{4}$ & $\frac{119}{540}$ & $\frac{35}{54}$ & $\frac{19}{54}$ & $\frac{31}{216}$ & $\frac{67}{810}$ & $\frac{191}{720}$ & $\frac{313}{6480}$ & $\frac{547}{6480}$ & $\frac{109}{720}$ & $\frac{59}{405}$ & $\frac{17}{216}$ \\
\hline$P_{i j}\left(F=27, I=1, S=\frac{1}{2}\right)$ & $\frac{1}{10}$ & $\frac{13}{30}$ & $\frac{7}{30}$ & $\frac{7}{30}$ & $\frac{2}{3}$ & $\frac{1}{3}$ & $\frac{37}{540}$ & $\frac{83}{540}$ & $\frac{79}{432}$ & $\frac{211}{2160}$ & $\frac{209}{2160}$ & $\frac{77}{432}$ & $\frac{41}{270}$ & $\frac{19}{270}$ \\
\hline$P_{i j}\left(F=35, I=1, S=\frac{1}{2}\right)$ & $\frac{1}{12}$ & $\frac{47}{108}$ & $\frac{1}{4}$ & $\frac{25}{108}$ & $\frac{16}{27}$ & $\frac{11}{27}$ & $\frac{433}{8640}$ & $\frac{2057}{8640}$ & $\frac{23}{192}$ & $\frac{1331}{8640}$ & $\frac{2207}{25920}$ & $\frac{11}{64}$ & $\frac{3469}{25920}$ & $\frac{407}{8640}$ \\
\hline$P_{i j}\left(F=\overline{35}, I=1, S=\frac{1}{2}\right)$ & $\frac{1}{12}$ & $\frac{47}{108}$ & $\frac{1}{4}$ & $\frac{25}{108}$ & $\frac{16}{27}$ & $\frac{11}{27}$ & $\frac{107}{1728}$ & $\frac{1481}{5184}$ & $\frac{107}{576}$ & $\frac{955}{5184}$ & $\frac{95}{1728}$ & $\frac{61}{576}$ & $\frac{149}{1728}$ & $\frac{61}{1728}$ \\
\hline$P_{i j}\left(F=1, I=0, S=\frac{9}{2}\right)$ & 0 & $\frac{3}{5}$ & $\frac{2}{5}$ & 0 & 1 & 0 & 0 & $\frac{1}{9}$ & $\frac{4}{9}$ & 0 & 0 & $\frac{2}{9}$ & $\frac{2}{9}$ & 0 \\
\hline$P_{i j}\left(F=8, I=0, S=\frac{7}{2}\right)$ & 0 & $\frac{3}{5}$ & $\frac{2}{5}$ & 0 & 1 & 0 & $\frac{7}{144}$ & $\frac{5}{48}$ & $\frac{7}{24}$ & $\frac{5}{72}$ & $\frac{7}{72}$ & $\frac{5}{24}$ & $\frac{7}{48}$ & $\frac{5}{144}$ \\
\hline$P_{i j}\left(F=1, I=0, S=\frac{5}{2}\right)$ & $\frac{4}{45}$ & $\frac{7}{15}$ & $\frac{14}{45}$ & $\frac{2}{15}$ & $\frac{7}{9}$ & $\frac{2}{9}$ & $\frac{8}{81}$ & $\frac{7}{81}$ & $\frac{28}{81}$ & $\frac{2}{81}$ & $\frac{4}{81}$ & $\frac{14}{81}$ & $\frac{14}{81}$ & $\frac{4}{81}$ \\
\hline$P_{i j}\left(F=8, I=0, S=\frac{5}{2}\right)$ & $\frac{91}{900}$ & $\frac{29}{60}$ & $\frac{269}{900}$ & $\frac{7}{60}$ & $\frac{83}{90}$ & $\frac{7}{90}$ & $\frac{91}{1620}$ & $\frac{71}{648}$ & $\frac{1841}{6480}$ & $\frac{83}{1296}$ & $\frac{581}{6480}$ & $\frac{263}{1296}$ & $\frac{497}{3240}$ & $\frac{13}{324}$ \\
\hline$P_{i j}\left(F=27, I=0, S=\frac{5}{2}\right)$ & $\frac{2}{75}$ & $\frac{8}{15}$ & $\frac{28}{75}$ & $\frac{1}{15}$ & $\frac{4}{5}$ & $\frac{1}{5}$ & $\frac{16}{405}$ & $\frac{14}{81}$ & $\frac{64}{405}$ & $\frac{11}{81}$ & $\frac{44}{405}$ & $\frac{16}{81}$ & $\frac{56}{405}$ & $\frac{4}{81}$ \\
\hline$P_{i j}\left(F=1, I=0, S=\frac{3}{2}\right)$ & $\frac{7}{60}$ & $\frac{17}{40}$ & $\frac{17}{60}$ & $\frac{7}{40}$ & $\frac{17}{24}$ & $\frac{7}{24}$ & $\frac{7}{54}$ & $\frac{17}{216}$ & $\frac{17}{54}$ & $\frac{7}{216}$ & $\frac{7}{108}$ & $\frac{17}{108}$ & $\frac{17}{108}$ & $\frac{7}{108}$ \\
\hline$P_{i j}\left(F=8, I=0, S=\frac{3}{2}\right)$ & $\frac{11}{150}$ & $\frac{1}{2}$ & $\frac{49}{150}$ & $\frac{1}{10}$ & $\frac{13}{15}$ & $\frac{2}{15}$ & $\frac{119}{1080}$ & $\frac{17}{216}$ & $\frac{497}{2160}$ & $\frac{41}{432}$ & $\frac{287}{2160}$ & $\frac{71}{432}$ & $\frac{119}{1080}$ & $\frac{17}{216}$ \\
\hline$P_{i j}\left(F=27, I=0, S=\frac{3}{2}\right)$ & $\frac{181}{2100}$ & $\frac{19}{40}$ & $\frac{659}{2100}$ & $\frac{1}{8}$ & $\frac{677}{840}$ & $\frac{163}{840}$ & $\frac{137}{2835}$ & $\frac{9}{56}$ & $\frac{47}{315}$ & $\frac{671}{4536}$ & $\frac{671}{5670}$ & $\frac{47}{252}$ & $\frac{9}{70}$ & $\frac{137}{2268}$ \\
\hline$P_{i j}\left(F=64, I=0, S=\frac{3}{2}\right)$ & $\frac{2}{35}$ & $\frac{7}{15}$ & $\frac{12}{35}$ & $\frac{2}{15}$ & $\frac{13}{21}$ & $\frac{8}{21}$ & $\frac{1}{112}$ & $\frac{107}{336}$ & $\frac{3}{56}$ & $\frac{41}{168}$ & $\frac{41}{504}$ & $\frac{9}{56}$ & $\frac{107}{1008}$ & $\frac{3}{112}$ \\
\hline$P_{i j}\left(F=8, I=0, S=\frac{1}{2}\right)$ & $\frac{3}{20}$ & $\frac{5}{12}$ & $\frac{1}{4}$ & $\frac{11}{60}$ & $\frac{5}{6}$ & $\frac{1}{6}$ & $\frac{7}{72}$ & $\frac{5}{54}$ & $\frac{35}{144}$ & $\frac{35}{432}$ & $\frac{49}{432}$ & $\frac{25}{144}$ & $\frac{7}{54}$ & $\frac{5}{72}$ \\
\hline$P_{i j}\left(F=27, I=0, S=\frac{1}{2}\right)$ & $\frac{2}{15}$ & $\frac{2}{5}$ & $\frac{4}{15}$ & $\frac{1}{5}$ & $\frac{2}{3}$ & $\frac{1}{3}$ & $\frac{4}{81}$ & $\frac{5}{27}$ & $\frac{4}{27}$ & $\frac{10}{81}$ & $\frac{8}{81}$ & $\frac{5}{27}$ & $\frac{4}{27}$ & $\frac{5}{81}$ \\
\hline
\end{tabular}

TABLE IX. Same as Table V for $q^{5} s^{4}$.

\begin{tabular}{|c|c|c|c|c|c|c|c|c|c|c|c|c|c|c|}
\hline \multirow{2}{*}{$\frac{q^{5} s^{4}}{\text { Flavor }}$} & \multicolumn{4}{|c|}{$i, j=1-5$} & \multicolumn{2}{|c|}{$i, j=6-9$} & \multicolumn{8}{|c|}{$i=1-5, j=6-9$} \\
\hline & $A$ & $S$ & $A$ & $S$ & $S$ & $S$ & $A$ & $S$ & $A$ & $S$ & A & $S$ & A & $S$ \\
\hline Color & $A$ & $A$ & $S$ & $S$ & $S$ & $A$ & A & A & $S$ & $S$ & $S$ & $S$ & $A$ & $A$ \\
\hline Spin & $A$ & $S$ & $S$ & $A$ & $A$ & $S$ & $A$ & $S$ & $S$ & $A$ & A & $S$ & $S$ & $A$ \\
\hline$P_{i j}\left(F=64, I=\frac{5}{2}, S=\frac{3}{2}\right)$ & 0 & $\frac{3}{5}$ & 0 & $\frac{2}{5}$ & $\frac{2}{3}$ & $\frac{1}{3}$ & $\frac{63}{1600}$ & $\frac{247}{1600}$ & $\frac{189}{800}$ & $\frac{57}{800}$ & $\frac{63}{800}$ & $\frac{171}{800}$ & $\frac{273}{1600}$ & $\frac{57}{1600}$ \\
\hline$P_{i j}\left(F=\overline{35}, I=\frac{5}{2}, S=\frac{1}{2}\right)$ & 0 & $\frac{3}{5}$ & 0 & $\frac{2}{5}$ & $\frac{2}{3}$ & $\frac{1}{3}$ & $\frac{9}{100}$ & $\frac{1}{10}$ & $\frac{27}{100}$ & $\frac{3}{50}$ & $\frac{9}{100}$ & $\frac{9}{50}$ & $\frac{3}{20}$ & $\frac{3}{50}$ \\
\hline$P_{i j}\left(F=27, I=\frac{3}{2}, S=\frac{5}{2}\right)$ & $\frac{1}{20}$ & $\frac{11}{20}$ & $\frac{1}{5}$ & $\frac{1}{5}$ & $\frac{2}{3}$ & $\frac{1}{3}$ & $\frac{211}{4800}$ & $\frac{659}{4800}$ & $\frac{23}{80}$ & $\frac{53}{1200}$ & $\frac{67}{1200}$ & $\frac{17}{80}$ & $\frac{901}{4800}$ & $\frac{149}{4800}$ \\
\hline$P_{i j}\left(F=\overline{10}, I=\frac{3}{2}, S=\frac{3}{2}\right)$ & $\frac{1}{16}$ & $\frac{25}{48}$ & $\frac{3}{16}$ & $\frac{11}{48}$ & $\frac{23}{36}$ & $\frac{13}{36}$ & $\frac{25}{256}$ & $\frac{25}{256}$ & $\frac{75}{256}$ & $\frac{11}{256}$ & $\frac{55}{768}$ & $\frac{45}{256}$ & $\frac{125}{768}$ & $\frac{15}{256}$ \\
\hline$P_{i j}\left(F=27, I=\frac{3}{2}, S=\frac{3}{2}\right)$ & $\frac{43}{560}$ & $\frac{39}{80}$ & $\frac{97}{560}$ & $\frac{21}{80}$ & $\frac{17}{28}$ & $\frac{11}{28}$ & $\frac{9743}{134400}$ & $\frac{17807}{134400}$ & $\frac{6961}{26880}$ & $\frac{6581}{134400}$ & $\frac{1237}{19200}$ & $\frac{737}{3840}$ & $\frac{3439}{19200}$ & $\frac{991}{19200}$ \\
\hline$P_{i j}\left(F=64, I=\frac{3}{2}, S=\frac{3}{2}\right)$ & $\frac{1}{28}$ & $\frac{31}{60}$ & $\frac{3}{14}$ & $\frac{7}{30}$ & $\frac{37}{63}$ & $\frac{26}{63}$ & $\frac{19}{700}$ & $\frac{533}{2100}$ & $\frac{57}{350}$ & $\frac{143}{1050}$ & $\frac{11}{150}$ & $\frac{9}{50}$ & $\frac{41}{300}$ & $\frac{3}{100}$ \\
\hline$P_{i j}\left(F=27, I=\frac{3}{2}, S=\frac{1}{2}\right)$ & $\frac{7}{80}$ & $\frac{39}{80}$ & $\frac{13}{80}$ & $\frac{21}{80}$ & $\frac{5}{8}$ & $\frac{3}{8}$ & $\frac{349}{3840}$ & $\frac{2189}{19200}$ & $\frac{4619}{19200}$ & $\frac{259}{3840}$ & $\frac{341}{3840}$ & $\frac{3421}{19200}$ & $\frac{2971}{19200}$ & $\frac{251}{3840}$ \\
\hline$P_{i j}\left(F=35, I=\frac{3}{2}, S=\frac{1}{2}\right)$ & $\frac{1}{10}$ & $\frac{7}{15}$ & $\frac{3}{20}$ & $\frac{17}{60}$ & $\frac{11}{18}$ & $\frac{7}{18}$ & $\frac{11}{200}$ & $\frac{43}{240}$ & $\frac{57}{320}$ & $\frac{499}{4800}$ & $\frac{421}{4800}$ & $\frac{63}{320}$ & $\frac{37}{240}$ & $\frac{9}{200}$ \\
\hline$P_{i j}\left(F=\overline{35}, I=\frac{3}{2}, S=\frac{1}{2}\right)$ & $\frac{1}{16}$ & $\frac{119}{240}$ & $\frac{3}{16}$ & $\frac{61}{240}$ & $\frac{43}{72}$ & $\frac{29}{72}$ & $\frac{469}{6400}$ & $\frac{269}{1280}$ & $\frac{1407}{6400}$ & $\frac{851}{6400}$ & $\frac{1327}{19200}$ & $\frac{873}{6400}$ & $\frac{433}{3840}$ & $\frac{291}{6400}$ \\
\hline$P_{i j}\left(F=8, I=\frac{1}{2}, S=\frac{7}{2}\right)$ & 0 & $\frac{3}{5}$ & $\frac{2}{5}$ & 0 & $\frac{2}{3}$ & $\frac{1}{3}$ & $\frac{3}{64}$ & $\frac{39}{320}$ & $\frac{11}{32}$ & $\frac{3}{160}$ & $\frac{1}{32}$ & $\frac{33}{160}$ & $\frac{13}{64}$ & $\frac{9}{320}$ \\
\hline$P_{i j}\left(F=8, I=\frac{1}{2}, S=\frac{5}{2}\right)$ & $\frac{21}{200}$ & $\frac{99}{200}$ & $\frac{59}{200}$ & $\frac{21}{200}$ & $\frac{2}{3}$ & $\frac{1}{3}$ & $\frac{11}{160}$ & $\frac{87}{800}$ & $\frac{103}{320}$ & $\frac{51}{1600}$ & $\frac{17}{320}$ & $\frac{309}{1600}$ & $\frac{29}{160}$ & $\frac{33}{800}$ \\
\hline$P_{i j}\left(F=27, I=\frac{1}{2}, S=\frac{5}{2}\right)$ & $\frac{1}{50}$ & $\frac{29}{50}$ & $\frac{19}{50}$ & $\frac{1}{50}$ & $\frac{2}{3}$ & $\frac{1}{3}$ & $\frac{1}{24}$ & $\frac{389}{2400}$ & $\frac{7}{32}$ & $\frac{59}{600}$ & $\frac{11}{120}$ & $\frac{153}{800}$ & $\frac{71}{480}$ & $\frac{29}{600}$ \\
\hline$P_{i j}\left(F=8, I=\frac{1}{2}, S=\frac{3}{2}\right)$ & $\frac{21}{200}$ & $\frac{89}{200}$ & $\frac{59}{200}$ & $\frac{31}{200}$ & $\frac{7}{12}$ & $\frac{5}{12}$ & $\frac{69}{640}$ & $\frac{333}{3200}$ & $\frac{181}{640}$ & $\frac{117}{3200}$ & $\frac{39}{640}$ & $\frac{543}{3200}$ & $\frac{111}{640}$ & $\frac{207}{3200}$ \\
\hline$P_{i j}\left(F=10, I=\frac{1}{2}, S=\frac{3}{2}\right)$ & $\frac{11}{80}$ & $\frac{109}{240}$ & $\frac{21}{80}$ & $\frac{7}{48}$ & $\frac{47}{72}$ & $\frac{25}{72}$ & $\frac{89}{1280}$ & $\frac{169}{1280}$ & $\frac{309}{1280}$ & $\frac{101}{1280}$ & $\frac{313}{3840}$ & $\frac{243}{1280}$ & $\frac{121}{768}$ & $\frac{63}{1280}$ \\
\hline$P_{i j}\left(F=27, I=\frac{1}{2}, S=\frac{3}{2}\right)$ & $\frac{281}{2800}$ & $\frac{1389}{2800}$ & $\frac{839}{2800}$ & $\frac{291}{2800}$ & $\frac{37}{56}$ & $\frac{19}{56}$ & $\frac{19}{336}$ & $\frac{5191}{33600}$ & $\frac{137}{672}$ & $\frac{3559}{33600}$ & $\frac{691}{6720}$ & $\frac{193}{1050}$ & $\frac{919}{6720}$ & $\frac{937}{16800}$ \\
\hline$P_{i j}\left(F=64, I=\frac{1}{2}, S=\frac{3}{2}\right)$ & $\frac{2}{35}$ & $\frac{53}{105}$ & $\frac{12}{35}$ & $\frac{2}{21}$ & $\frac{38}{63}$ & $\frac{25}{63}$ & $\frac{53}{2240}$ & $\frac{2039}{6720}$ & $\frac{159}{1120}$ & $\frac{713}{3360}$ & $\frac{239}{3360}$ & $\frac{153}{1120}$ & $\frac{593}{6720}$ & $\frac{51}{2240}$ \\
\hline$P_{i j}\left(F=8, I=\frac{1}{2}, S=\frac{1}{2}\right)$ & $\frac{7}{40}$ & $\frac{47}{120}$ & $\frac{9}{40}$ & $\frac{5}{24}$ & $\frac{11}{18}$ & $\frac{7}{18}$ & $\frac{7}{64}$ & $\frac{31}{320}$ & $\frac{9}{32}$ & $\frac{7}{160}$ & $\frac{7}{96}$ & $\frac{27}{160}$ & $\frac{31}{192}$ & $\frac{21}{320}$ \\
\hline$P_{i j}\left(F=27, I=\frac{1}{2}, S=\frac{1}{2}\right)$ & $\frac{13}{80}$ & $\frac{33}{80}$ & $\frac{19}{80}$ & $\frac{3}{16}$ & $\frac{5}{8}$ & $\frac{3}{8}$ & $\frac{49}{768}$ & $\frac{611}{3840}$ & $\frac{151}{768}$ & $\frac{389}{3840}$ & $\frac{71}{768}$ & $\frac{709}{3840}$ & $\frac{113}{768}$ & $\frac{211}{3840}$ \\
\hline$P_{i j}\left(F=35, I=\frac{1}{2}, S=\frac{1}{2}\right)$ & $\frac{11}{80}$ & $\frac{101}{240}$ & $\frac{21}{80}$ & $\frac{43}{240}$ & $\frac{43}{72}$ & $\frac{29}{71}$ & $\frac{5}{128}$ & $\frac{433}{1920}$ & $\frac{87}{640}$ & $\frac{287}{1920}$ & $\frac{173}{1920}$ & $\frac{117}{640}$ & $\frac{259}{1920}$ & $\frac{27}{640}$ \\
\hline
\end{tabular}


TABLE X. Same as Table V for $q^{4} s^{5}$.

\begin{tabular}{|c|c|c|c|c|c|c|c|c|c|c|c|c|c|c|}
\hline \multirow{2}{*}{$\frac{q^{4} s^{5}}{\text { Flavor }}$} & \multicolumn{4}{|c|}{$i, j=1-4$} & \multicolumn{2}{|c|}{$i, j=5-9$} & \multicolumn{8}{|c|}{$i=1-4, j=5-9$} \\
\hline & $A$ & $S$ & $A$ & $S$ & $S$ & $S$ & $A$ & $S$ & $A$ & $S$ & $A$ & $S$ & $A$ & $S$ \\
\hline Color & $A$ & $A$ & $S$ & $S$ & $S$ & $A$ & $A$ & $A$ & $S$ & $S$ & $S$ & $S$ & $A$ & $A$ \\
\hline Spin & $A$ & $S$ & $S$ & $A$ & $A$ & $S$ & $A$ & $S$ & $S$ & $A$ & $A$ & $S$ & $S$ & $A$ \\
\hline$P_{i j}\left(F=64, I=2, S=\frac{3}{2}\right)$ & 0 & $\frac{2}{3}$ & 0 & $\frac{1}{3}$ & $\frac{3}{5}$ & $\frac{2}{5}$ & $\frac{63}{1600}$ & $\frac{247}{1600}$ & $\frac{189}{800}$ & $\frac{57}{800}$ & $\frac{63}{800}$ & $\frac{171}{800}$ & $\frac{273}{1600}$ & $\frac{57}{1600}$ \\
\hline$P_{i j}\left(F=\overline{35}, I=2, S=\frac{1}{2}\right)$ & 0 & $\frac{2}{3}$ & 0 & $\frac{1}{3}$ & $\frac{3}{5}$ & $\frac{2}{5}$ & $\frac{9}{100}$ & $\frac{1}{10}$ & $\frac{27}{100}$ & $\frac{3}{50}$ & $\frac{9}{100}$ & $\frac{9}{50}$ & $\frac{3}{20}$ & $\frac{3}{50}$ \\
\hline$P_{i j}\left(F=27, I=1, S=\frac{5}{2}\right)$ & 0 & $\frac{2}{3}$ & $\frac{1}{3}$ & 0 & $\frac{3}{5}$ & $\frac{2}{5}$ & $\frac{3}{50}$ & $\frac{3}{25}$ & $\frac{3}{10}$ & $\frac{1}{25}$ & $\frac{3}{50}$ & $\frac{1}{5}$ & $\frac{9}{50}$ & $\frac{1}{25}$ \\
\hline$P_{i j}\left(F=27, I=1, S=\frac{3}{2}\right)$ & $\frac{5}{42}$ & $\frac{23}{42}$ & $\frac{3}{14}$ & $\frac{5}{42}$ & $\frac{3}{5}$ & $\frac{2}{5}$ & $\frac{213}{2800}$ & $\frac{153}{1400}$ & $\frac{159}{560}$ & $\frac{71}{1400}$ & $\frac{213}{2800}$ & $\frac{53}{280}$ & $\frac{459}{2800}$ & $\frac{71}{1400}$ \\
\hline$P_{i j}\left(F=64, I=1, S=\frac{3}{2}\right)$ & $\frac{1}{21}$ & $\frac{13}{21}$ & $\frac{2}{7}$ & $\frac{1}{21}$ & $\frac{3}{5}$ & $\frac{2}{5}$ & $\frac{387}{11200}$ & $\frac{2619}{11200}$ & $\frac{1161}{5600}$ & $\frac{177}{1120}$ & $\frac{87}{1120}$ & $\frac{879}{5600}$ & $\frac{1181}{11200}$ & $\frac{293}{11200}$ \\
\hline$P_{i j}\left(F=27, I=1, S=\frac{1}{2}\right)$ & $\frac{5}{24}$ & $\frac{11}{24}$ & $\frac{1}{8}$ & $\frac{5}{24}$ & $\frac{3}{5}$ & $\frac{2}{5}$ & $\frac{33}{400}$ & $\frac{21}{200}$ & $\frac{111}{400}$ & $\frac{11}{200}$ & $\frac{33}{400}$ & $\frac{37}{200}$ & $\frac{63}{400}$ & $\frac{11}{200}$ \\
\hline$P_{i j}\left(F=35, I=1, S=\frac{1}{2}\right)$ & $\frac{5}{24}$ & $\frac{11}{24}$ & $\frac{1}{8}$ & $\frac{5}{24}$ & $\frac{3}{5}$ & $\frac{2}{5}$ & $\frac{3}{64}$ & $\frac{51}{320}$ & $\frac{333}{1600}$ & $\frac{153}{1600}$ & $\frac{147}{1600}$ & $\frac{327}{1600}$ & $\frac{49}{320}$ & $\frac{13}{320}$ \\
\hline$P_{i j}\left(F=10, I=0, S=\frac{3}{2}\right)$ & $\frac{1}{4}$ & $\frac{5}{12}$ & $\frac{1}{4}$ & $\frac{1}{12}$ & $\frac{3}{5}$ & $\frac{2}{5}$ & $\frac{27}{400}$ & $\frac{23}{200}$ & $\frac{117}{400}$ & $\frac{9}{200}$ & $\frac{27}{400}$ & $\frac{39}{200}$ & $\frac{69}{400}$ & $\frac{9}{200}$ \\
\hline$P_{i j}\left(F=35, I=0, S=\frac{1}{2}\right)$ & $\frac{1}{4}$ & $\frac{5}{12}$ & $\frac{1}{4}$ & $\frac{1}{12}$ & $\frac{3}{5}$ & $\frac{2}{5}$ & $\frac{27}{800}$ & $\frac{143}{800}$ & $\frac{27}{160}$ & $\frac{99}{800}$ & $\frac{81}{800}$ & $\frac{33}{160}$ & $\frac{117}{800}$ & $\frac{33}{800}$ \\
\hline
\end{tabular}

where $m$ is the multiplicity of the corresponding state. We can also represent the orbital state of a tribaryon in terms of baryon configuration. There are four possible representations,

$$
\begin{aligned}
& \left|O_{1}\right\rangle=\left|\begin{array}{|l|l|l|}
\hline B_{1} & B_{2} & B_{3}
\end{array}\right\rangle, \quad\left|O_{2}\right\rangle=\left|\begin{array}{|l|l|}
\hline B_{1} & B_{2} \\
\hline B_{3} &
\end{array}\right\rangle, \\
& \left|O_{3}\right\rangle=\left|\begin{array}{|l|l|}
\hline B_{1} & B_{3} \\
\hline B_{2} &
\end{array}\right\rangle, \quad\left|O_{4}\right\rangle=\mid \begin{array}{|l|}
\hline B_{1} \\
\hline B_{2} \\
\hline B_{3}
\end{array} \\
& \hline
\end{aligned}
$$

where $B_{i}$ represents an orbital state of the $i$ th baryon. Now, $\left|O_{1}\right\rangle$ and $\left|O_{4}\right\rangle$ states are symmetric and antisymmetric under the exchange among three baryons, respectively.

On the other hand, $\left|O_{2}\right\rangle$ and $\left|O_{3}\right\rangle$ states have mixed symmetric property. By constructing the orbital state using the above representation, we can connect this representation to Eq. (A2). For [9], it is obvious that it corresponds to $\left|O_{1}\right\rangle$. For $[8,1]$, let us consider the following orbital basis set for $[8,1]$ :

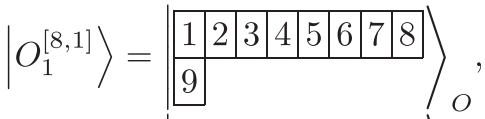

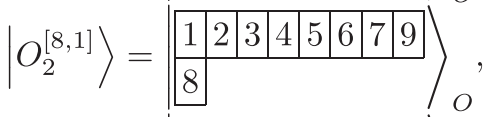

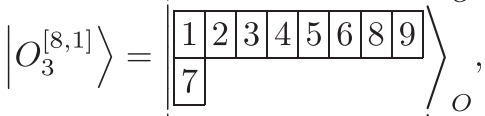

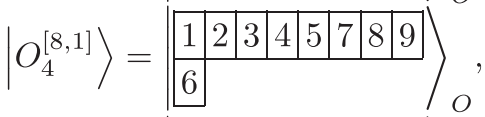

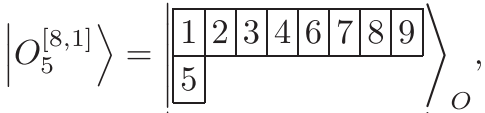

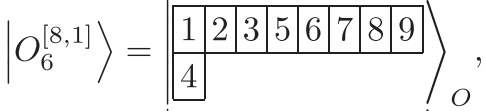

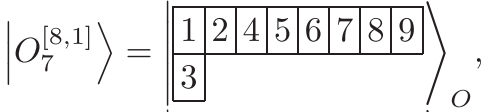

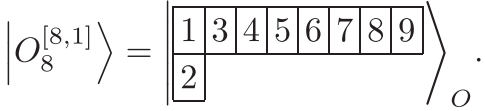

Then, we can construct $\left|O_{2}\right\rangle$ and $\left|O_{3}\right\rangle$ in Eq. (A3) using

\begin{tabular}{|c|c|c|c|c|c|c|c|c|c|c|c|c|c|c|}
\hline \multirow{2}{*}{$\frac{q^{3} s^{6}}{\text { Flavor }}$} & \multicolumn{4}{|c|}{$i, j=1-3$} & \multicolumn{2}{|c|}{$i, j=4-9$} & \multicolumn{8}{|c|}{$i=1-3, j=4-9$} \\
\hline & $A$ & $S$ & $A$ & $S$ & $S$ & $S$ & $A$ & $S$ & $A$ & $S$ & $A$ & $S$ & $A$ & $S$ \\
\hline Color & $A$ & $A$ & $S$ & $S$ & $S$ & $A$ & $A$ & $A$ & $S$ & $S$ & $S$ & $S$ & $A$ & $A$ \\
\hline Spin & $A$ & $S$ & $S$ & $A$ & $A$ & $S$ & $A$ & $S$ & $S$ & $A$ & $A$ & $S$ & $S$ & $A$ \\
\hline$P_{i j}\left(F=64, I=\frac{3}{2}, S=\frac{3}{2}\right)$ & 0 & 1 & 0 & 0 & $\frac{3}{5}$ & $\frac{2}{5}$ & $\frac{7}{144}$ & $\frac{5}{48}$ & $\frac{7}{24}$ & $\frac{5}{72}$ & $\frac{7}{72}$ & $\frac{5}{24}$ & $\frac{7}{48}$ & $\frac{5}{144}$ \\
\hline$P_{i j}\left(F=35, I=\frac{1}{2}, S=\frac{1}{2}\right)$ & $\frac{1}{2}$ & $\frac{1}{2}$ & 0 & 0 & $\frac{3}{5}$ & $\frac{2}{5}$ & $\frac{7}{144}$ & $\begin{array}{l}40 \\
\frac{5}{48}\end{array}$ & $\begin{array}{l}\frac{24}{24} \\
-12\end{array}$ & $\frac{5}{72}$ & $\frac{7}{72}$ & $\frac{5}{24}$ & $\begin{array}{l}40 \\
\frac{7}{48}\end{array}$ & $\begin{array}{l}\frac{144}{144} \\
144\end{array}$ \\
\hline
\end{tabular}
the $[8,1]$ basis as follows:

TABLE XI. Same as Table V for $q^{3} s^{6}$. 
TABLE XII. The relation between three baryons and nine quarks configurations. $\bigcirc$ represents that there is a connection between the two configurations.

\begin{tabular}{lcccc}
\hline \hline & $O_{1}$ & $O_{2}$ & $O_{3}$ & $O_{4}$ \\
\hline$[9]$ & $\bigcirc$ & & & \\
{$[81]$} & & $\bigcirc$ & $\bigcirc$ & \\
{$[72]$} & 0 & $\bigcirc$ & $\bigcirc$ & \\
{$[63]$} & $\bigcirc$ & $\bigcirc$ & $\bigcirc$ & \\
{$[54]$} & & $\bigcirc$ & $\bigcirc$ & \\
{$[711]$} & & & & \\
{$[621]$} & & $\bigcirc$ & $\bigcirc$ & \\
{$[531]$} & & $\bigcirc$ & $\bigcirc$ & \\
{$[522]$} & & & & \\
{$[432]$} & & $\bigcirc$ & $\bigcirc$ & \\
{$[333]$} & & & & \\
\hline \hline
\end{tabular}

$$
\begin{aligned}
& \left|O_{2}\right\rangle=\frac{1}{2}\left|O_{1}^{[8,1]}\right\rangle+\frac{3}{2 \sqrt{7}}\left|O_{2}^{[8,1]}\right\rangle+\frac{\sqrt{3}}{\sqrt{7}}\left|O_{3}^{[8,1]}\right\rangle \\
& \left|O_{3}\right\rangle=\frac{1}{\sqrt{5}}\left|O_{4}^{[8,1]}\right\rangle+\frac{\sqrt{3}}{\sqrt{10}}\left|O_{5}^{[8,1]}\right\rangle+\frac{1}{\sqrt{2}}\left|O_{6}^{[8,1]}\right\rangle .
\end{aligned}
$$

We can check that $\left|\mathrm{O}_{2}\right\rangle$ is symmetric under (14)(25)(36) and $\left|\mathrm{O}_{3}\right\rangle$ is antisymmetric under (14)(25)(36). Also, the above states satisfy the other symmetric properties which the $[2,1]$ Young diagram has. Using similar method, we can find the relation between the two representations with other Young diagrams. The open circles in Table XII show the nonvanishing overlap between the two representations.
[1] A. De Rujula, H. Georgi, and S. L. Glashow, Phys. Rev. D 12, 147 (1975).

[2] L. Y. Glozman and D. O. Riska, Phys. Rep. 268, 263 (1996).

[3] R. L. Jaffe, Phys. Rev. Lett. 38, 195 (1977).

[4] R. L. Jaffe, Phys. Rev. D 15, 267 (1977).

[5] R. L. Jaffe, Phys. Rev. D 15, 281 (1977).

[6] S. Cho et al. (ExHIC Collaboration), Prog. Part. Nucl. Phys. 95, 279 (2017).

[7] Y. R. Liu, H. X. Chen, W. Chen, X. Liu, and S. L. Zhu, Prog. Part. Nucl. Phys. 107, 237 (2019).

[8] M. Oka and K. Yazaki, Prog. Theor. Phys. 66, 556 (1981).

[9] T. Inoue, N. Ishii, S. Aoki, T. Doi, T. Hatsuda, Y. Ikeda, K. Murano, H. Nemura, and K. Sasaki (HAL QCD Collaboration), Phys. Rev. Lett. 106, 162002 (2011).

[10] A. Park, S. H. Lee, T. Inoue, and T. Hatsuda, Eur. Phys. J. A 56, 93 (2020).
[11] Y. Maezawa, T. Hatsuda, and S. Sasaki, Prog. Theor. Phys. 114, 317 (2005).

[12] R. Tamagaki, Prog. Theor. Phys. 119, 965 (2008).

[13] H. Garcilazo and A. Valcarce, Phys. Rev. C 93, 034001 (2016).

[14] A. Park, W. Park, and S. H. Lee, Phys. Rev. D 98, 034001 (2018).

[15] A. Park and S. H. Lee, Phys. Rev. C 100, 055201 (2019).

[16] A. Park and S. H. Lee, J. Phys. Soc. Jpn. Conf. Proc. 26, 023019 (2019).

[17] J. M. Lattimer and M. Prakash, Phys. Rep. 442, 109 (2007).

[18] I. Bombaci, J. Phys. Soc. Jpn. Conf. Proc. 17, 101002 (2017).

[19] W. Park, A. Park, and S. H. Lee, Phys. Rev. D 93, 074007 (2016).

[20] F. Stancu, Oxford Stud. Nucl. Phys. 19, 1 (1996).

[21] A. Park, W. Park, and S. H. Lee, Phys. Rev. D 96, 034029 (2017). 